See discussions, stats, and author profiles for this publication at: https://www.researchgate.net/publication/330262214

\title{
Elastic properties of the TiZrNbTaMo multi-principal element alloy studied from first principles
}

Article in Intermetallics · March 2019

DOI: $10.1016 /$ j.intermet.2018.12.014

CITATIONS

4 authors, including:

Natalia Koval

CIC nanoGUNE Consolide

7 PUBLICATIONS 15 CITATIONS

SEE PROFILE

7 Ricardo Diez Muino

Centro de Física de Materiales, Centro Mixto CSIC-UPV/EHU

109 PUBLICATIONS 2,207 CITATIONS

SEE PROFILE

Some of the authors of this publication are also working on these related projects:

Project Reactive scattering of $\mathrm{HCl}$ on $\mathrm{Au}(111)$ View project

Project Radiation damage in materials relevant to space exploration View project
READS

30

J. I. Juaristi

Universidad del País Vasco / Euskal Herriko Unibertsitatea

133 PUBLICATIONS 1,611 CITATIONS

SEE PROFILE 


\title{
Elastic properties of the TiZrNbTaMo multi-principal element alloy studied from first-principles
}

\author{
Natalia E. Koval ${ }^{\mathrm{a}, \mathrm{b}, *}$, Joseba Iñaki Juaristi ${ }^{\mathrm{c}, \mathrm{a}, \mathrm{b}}$, Ricardo Díez Muiño ${ }^{\mathrm{a}, \mathrm{b}}$, Maite \\ Alducin $^{\mathrm{a}, \mathrm{b}}$ \\ ${ }^{a}$ Donostia International Physics Center DIPC, Paseo Manuel de Lardizabal 4, \\ 20018 Donostia-San Sebastián, Spain \\ ${ }^{b}$ Centro de Física de Materiales CFM/MPC (CSIC-UPV/EHU), Paseo Manuel de \\ Lardizabal 5, 20018 Donostia-San Sebastián, Spain \\ ${ }^{c}$ Departamento de Física de Materiales, Facultad de Químicas (UPV/EHU), \\ Apartado 1072, 20080 Donostia-San Sebastián, Spain
}

\begin{abstract}
The TiZrNbTaMo multi-principal element alloy has been postulated as a new potential material for biomedical applications, such as orthopedic implants. Besides the good biocompatibility of the constituent atoms, the TiZrNbTaMo alloy also exhibits excellent corrosion resistance and mechanical properties, according to recent experimental studies. Motivated by these experiments, here we investigate with density functional theory (DFT) the structure, as well as the elastic and electronic properties, of the equiatomic and nearly-equiatomic TiZrNbTaMo alloys. By combining evolutionary algorithms with DFT calculations of the energy, we can correctly predict the crystal structures of the two phases that are identified in experiments. The corresponding elastic properties, which are also calculated with DFT, are in good qualitative agreement with the experimental observations. The analysis of the electronic properties allows us to explain the differences in the elastic moduli between the two phases in terms of the differences in both the electron density distribution and the bonding-states occupation.
\end{abstract}

Keywords: A. high-entropy alloys; B. elastic properties, biocompatibility,

\footnotetext{
* Corresponding author

Email addresses: natalia_koval@ehu.eus (Natalia E. Koval), josebainaki.juaristi@ehu.eus (Joseba Iñaki Juaristi), rdm@ehu.eus (Ricardo Díez Muiño), maite.alducin@ehu.eus (Maite Alducin)
}

Preprint submitted to Intermetallics

17 December 2018 
density functional theory; E. ab-initio calculations, electronic structure, calculation

\section{Introduction}

Multi-component alloys, also known as multi-principal element alloys (MPEAs) [1, or complex concentrated alloys (CCAs) 2], have received growing attention in the last decade due to the recently emerged concept of the so-called high-entropy alloys (HEAs) [3. By definition HEAs are composed of at least five metallic elements in equiatomic or nearly equiatomic proportions. The name "highentropy alloy" comes from the fact that random solid solutions of these alloys have considerably higher mixing entropy than the conventional alloys 4 . The senary and quinary equiatomic $\mathrm{CuCoNiCrAlFe} \mathrm{[4}$ and $\mathrm{CoCrFeMnNi} \mathrm{[5]} \mathrm{alloys}$ were the first single-phase HEAs reported in the literature. The mere existence of a single-phase multi-component alloy that, in addition, exhibited superior mechanical properties clearly clashed with the prevailing models describing the formation of conventional alloys. Specifically, the existing binary and ternary phase diagrams predicted that multi-component alloys may form many different kinds of phases and intermetallic compounds, resulting in complicated and brittle microstructures without any practical relevance [6]. However, in spite of these expectations, the extensive experimental work performed along the last decade has demonstrated the existence of many other HEAs characterized by a single-phase solid solution and promising engineering properties [2, 3, 7, , 8, 9,

All these findings suggested that the high configurational entropy, achieved by adding more alloying elements, plays an important role in the stabilization of single-phase solid solutions with simple crystal structures, being mostly body-centered cubic (bcc) and face-centered cubic (fcc), and less often hexagonal closed-packed (hcp) 2. More recent studies, however, show that the high configurational entropy is neither a sufficient nor a necessary condition to form single-phase solid solution [10, 11]. Many MPEAs consisting of five or more elements are multi-phase, i.e., do not form a single-phase solid solution structure, 
while still have good mechanical properties. In the light of these new observations, the authors suggest to distinguish between the high-entropy alloys, which exhibit single-phase solid solution microstructures, and the multi-component alloys, which form multi-phase structures [11. The identification of the singlephase or multi-phase character is done experimentally, but recent theoretical advances in the search for simple and reliable criteria that can predict the HEA or MPEA formation are quite promising [12.

The interest in studying refractory MPEAs is growing due to their promising mechanical properties, such as high strength and ductility [13, 14, 15, 16, 17, 18, as well as their good biocompatibility allowing to use these alloys for biomedical applications [19, 20, 21, 22, 23, 24, 25, 26]. In particular, the TiZrNbTaMo alloy has been experimentally investigated for its possible application as a metallic biomaterial in Refs. [27, 28. In both cases, the as-cast TiZrNbTaMo alloy is observed to form a dendritic two-phase structure, in which dendritic arms and interdendritic regions are two different bcc phases with nearly equiatomic composition [27, 28] and, therefore, we will refer to it as a MPEA in the following. Important to us, the authors find that this alloy exhibits much higher corrosion resistance than the conventional CoCrMo and 316LSS alloys used for bone implants, as well as high yield strength, with a Vickers microhardness of $4.9 \mathrm{GPa}$ and Young's modulus of $153 \mathrm{GPa}$. This value of Young's modulus is lower than the values for CoCrMo (240 GPa) and 316LSS (193 GPa) alloys and thus is closer to that of bones 29 .

Motivated by these findings, we study and characterize from first principles the structure, elastic properties, and electronic properties of the equiatomic and nearly-equiatomic TiZrNbTaMo MPEAs. In this respect, our final objective is to correlate the mechanical properties specific to each phase with their corresponding electronic structure. However, we should first be able to determine theoretically the structure of the TiZrNbTaMo MPEAs that are reported experimentally.

Currently, most of the theoretical studies on HEAs and MPEAs are using two methodologies to describe the structure of disordered alloys, namely, the 
coherent potential approximation (CPA) [30, 31, 32, 33] and the special quasirandom structures (SQS) approximation [34, 35, 36, 37. Both methods have been undoubtedly useful in describing the structure of MPEAs in which the atoms are randomly distributed, but the two of them require the knowledge of the crystal structure. In order to overcome this limitation, which restricts the predictability of these approaches, another objective of our present work is to explore a different approach for predicting the structures of alloys that do not require the knowledge of any experimental parameters, such as crystal symmetry and lattice constant. The method we use is based on density functional theory (DFT) calculations of the energy and the concept of the Darwinian evolution to select the best structures. Thus, the alloy structure is fully obtained from first principles. This methodology is implemented in the Universal Structure Predictor: Evolutionary Xtallography (USPEx) code [38, 39, 40] and it has been successfully applied to predict the structure of nanoparticles, polymers, 2D-crystals, surfaces, and interfaces at different temperature and pressure conditions [41]. In the present work, we show that this methodology is also able to predict the bcc structure of the TiZrNbTaMo MPEA.

The paper is organized as follows: in Sec. 2 we briefly describe the USPEX method, as well as the details of the DFT calculations of the elastic properties. The results are discussed in Sec. 3 . We start the section by comparing the TiZrNbTa and TiZrNbTaMo alloys in an attempt to identify the role of Mo in improving the elastic properties. Next, we present the predicted structures for the different alloys and the corresponding calculations of their elastic moduli, while comparing them to the experimental data. We end Sec. 3 by analyzing the effect of the electronic structure on the elastic properties. Finally, the conclusions are summarized in Sec. 4 


\section{Computational methods}

\subsection{Evolutionary algorithm for crystal structure prediction}

Finding the stable structure of a compound knowing only its chemical composition is a long-standing problem in theoretical condensed matter physics and chemistry. Recent advances in evolutionary algorithms help to advance in the solution of this problem. Here we use the crystal structure prediction code known as USPEX [38, 39, 40]. This package, which allows combining DFT calculations of the system properties with the concept of an evolutionary selection among all possible structures, does not require any system-specific knowledge except for the chemical composition. In this respect, the system structure is fully derived from first principles. Briefly, the structure search procedure is as follows. The algorithm starts creating crystal structures in the first generation by a random-number generator. These structures have to satisfy a number of "hard constraints", such as the minimum acceptable interatomic distance, the minimum value of a lattice parameter, and the minimum and maximum cell angles (from $60^{\circ}$ to $120^{\circ}$ ) [38. For each structure, the energy is calculated using an external solver. In this work, all the calculations of the energies are carried out using the Vienna ab initio simulation package (VASP) 42, 43], a DFT-based code with plane-wave basis sets. A set of structures with the lowest ground state energies in each generation is used to produce new structures in the next generation by means of the evolutionary techniques, such as heredity (from two "parent" structures), permutation between atoms of a different type, lattice mutation, and rotation. In our calculations, we mostly used the permutation technique, with a small percentage of heredity and atomic mutations, since our aim is to obtain the most disordered structure. The process is repeated until the global minimum is reached, i.e., when the structure with minimum energy is repeatedly found in a finite number of generations that is defined as an input parameter.

As described in sec. 3.1, we additionally use the so-called quasi-random structure (QRS) technique implemented in the USPEX code for some of the studied 
alloys. This technique is similar to the SQS method and is based on the search of the most structurally disordered state for a given initial structure. The algorithm minimizes the structural order in the system [44, which is a parameter related to the pair correlation function, by permutation of atoms in the cell. Thus, only the pair correlation function is used to describe the random disorder in the QRS structural model.

For the sake of completeness, the results predicted by the evolutionary algorithm will be contrasted to the valence electron concentration (VEC) predictions. The latter is a simple empirical criterion that is commonly used to predict the stability of fcc and bcc solid solutions with quite remarkable success [45]. The average VEC of the alloy is calculated from the VECs of all constituent elements as $\mathrm{VEC}=\sum_{i} c_{i}(\mathrm{VEC})_{i}$, where $c_{i}$ is the atomic percentage of the $i$-th element and $(\mathrm{VEC})_{i}$ is the number of valence electrons of the $i$-th element (including outer $d$-electrons). The fcc phase - or a combination of fcc phases [3]is found to be stable for alloys with $\mathrm{VEC} \geq 8$, while the bcc phase -or a combination of bcc phases [3] - is stable at low VEC $<6.87$. The combination of bcc and fcc phases is usually observed in the case of intermediate VEC values.

Once the structures of the desired alloys are found by USPEx, we calculate with DFT their elastic properties, as well as their electronic properties. The next section summarizes how to calculate the different elastic moduli from the single-crystal elastic constants.

\subsection{Calculation of the elastic properties}

The elastic properties of the alloys are studied within DFT by the stressstrain approach of Le Page and Saxe [46], which is implemented in VASP. This approach is based on the Hooke's law for elastic solids, which describes the linear dependence between the applied external stress components $\sigma_{i}(i=1,6)$ and the small strain $\epsilon_{j}(j=1,6)$ caused in the relaxed crystal as, 47, 48]

$$
\sigma_{i}=\sum_{j=1,6} c_{i j} \epsilon_{j}
$$


Here $c_{i j}$ are the single-crystal elastic (stiffness) constants of the material, which are represented by a $6 \times 6$ tensor. Following Ref. [46, the total elastic tensor is obtained by performing six finite distortions of the equilibrium lattice configuration.

In a system with cubic symmetry, there are only three independent elastic constants, namely, $c_{11}, c_{12}$, and $c_{44}$ [47, 48, 49]. In our case, however, the structures of the alloys under consideration are slightly distorted because of the sizable differences that exist among the bond lengths of the constituent elements. As a result, there are nine non-identical elastic constants: $c_{11}, c_{12}$, $c_{13}, c_{23}, c_{22}, c_{33}, c_{44}, c_{55}$, and $c_{66}$. Thus, in order to obtain the cubic elastic constants of our distorted bcc structures, we follow the averaging scheme [50, 51] and redefine the three independent elastic constants as, $C_{11}=\left(c_{11}+c_{22}+c_{33}\right) / 3$, $C_{12}=\left(c_{12}+c_{13}+c_{23}\right) / 3$, and $C_{44}=\left(c_{44}+c_{55}+c_{66}\right) / 3$.

Once the theoretical elastic constants of the single-crystal structure are known, we adopt the Voight-Reuss-Hill approximation to obtain the elastic properties of a polycrystalline material, such as the bulk modulus $B$, the shear modulus $G$, the Young's modulus $E$, and the Poisson's ratio $\nu$, as an average of the single-crystal values over all orientations [52]. In doing this average, the approximation by Voigt [53] assumes that the strain is uniform throughout the material, while the approximation by Reuss [54] assumes a uniform stress. As a result, the obtained elastic moduli may depend on the approximation used. Next, we briefly summarize the expressions and meaning of the elastic moduli calculated in the present work.

The Voight and Reuss expressions for the bulk modulus, which describes the resistance to volume change under external pressure, are the same in both approximations and read [52]:

$$
B=\left(C_{11}+2 C_{12}\right) / 3 \text {. }
$$

In the case of the shear modulus, which describes the elastic properties of a solid under the application of transverse forces such as shearing or torsion, the 
Reuss and Voight expressions are, respectively,

$$
G_{R}=\frac{5\left(C_{11}-C_{12}\right) C_{44}}{4 C_{44}+3\left(C_{11}-C_{12}\right)} ; G_{V}=\frac{\left(C_{11}-C_{12}+3 C_{44}\right)}{5} .
$$

Compared to the experimental values, the Reuss $G_{R}$ and Voight $G_{V}$ shear modulus usually represent the lower and upper limits, respectively. Thus, a common practice is the use of their corresponding mean value as suggested by Hill [55],

$$
G=\left(G_{V}+G_{R}\right) / 2 .
$$

The stiffness of a solid material is characterized by the Young's or elastic modulus E. Specifically, Young's modulus measures the resistance of a material to elastic (recoverable) deformation under load. Finally, another property of interest is the Poisson's ratio $\nu$, defined as the ratio of the relative contraction strain normal to the applied force to the relative extension strain in the direction of the applied force. Both, $E$ and $\nu$ can be obtained from the bulk and the (mean) shear moduli as

$$
E=\frac{9 B G}{3 B+G} \quad \text { and } \quad \nu=\frac{3 B-2 G}{2(3 B+G)} .
$$

In using Eqs. (3)-(5), it is important to remember that their applicability becomes increasingly invalid in the case of rather anisotropic polycrystalline materials [52. In this respect, there are two variables that serve to characterize the elastic anisotropy of the system. The Zener anisotropy ratio $A_{Z}=2 C_{44} /\left(C_{11}-C_{12}\right)[56$ should take values close to unity for an elastically isotropic material. Another measure of the anisotropy is the ratio $A_{G_{V R}}=$ $\left(G_{V}-G_{R}\right) /\left(G_{V}+G_{R}\right)$ that for elastically isotropic material should approach zero, meaning that both calculation methods (Voigt and Reuss) give similar results. In all the alloys studied in this work, we also verify the values for these two elastic anisotropy ratios in order to check the reliability of the theoretical elastic moduli.

\subsection{Computational settings}

All DFT calculations are performed within the generalized gradient approximation of Perdew-Burke-Ernzerhof (PBE) [57] to the exchange-correlation func- 
tional. The electron-core interaction is treated with the projector augmentedwave (PAW) method [58, using the implementation provided and developed for VASP [59. In particular, the number of valence electrons for each element is as follows, $\operatorname{Ti}\left(12 e^{-}\right), \operatorname{Zr}\left(12 e^{-}\right), \operatorname{Nb}\left(13 e^{-}\right), \operatorname{Ta}\left(11 e^{-}\right)$, and $\mathrm{Mo}\left(14 e^{-}\right)$. The accuracy of the calculations regarding the basis set and sampling of the Brillouin zone is adapted to the specificities of the property under consideration. In calculations related with the density of states (DOS), the one-electron wave functions are expanded in a plane-wave basis set with an energy cutoff of $600 \mathrm{eV}$, while the corresponding fractional occupancies are determined through the tetrahedron method with Blöchl corrections [60. For the usually sensitive calculations of the elastic tensor, the energy cutoff is increased to $700 \mathrm{eV}$. In this case, the Methfessel and Paxton broadening scheme of the first order [61] is used for the fractional occupancies. The minimum energy criterion for total energy self-consistency is $10^{-7} \mathrm{eV}$. In both types of calculations, the Brillouin zone integration is performed with a sufficiently large, $\Gamma$-centered, Monkhorst-Pack grid of special $\mathbf{k}$-points 62 . The total number of $\mathbf{k}$-points is adapted to the symmetry of the unit cell used in each of the studied alloys and it will be specified in the text.

Prior to starting the systematic search of the most stable crystal structure, we verify the quality of the computational settings in reproducing the lattice parameter $a$ (and also $c$ for the hcp crystals), cohesive energy $E_{\mathrm{coh}}$, and various elastic properties of the single-metal crystals for each of the elements forming the alloys. The values $E_{\text {coh }}$ include the correction to the individual atomic energies obtained from a spin-polarized calculation. Our values are in agreement with those calculated by other authors using the PBE exchange-correlation functional [63, 64. The results are summarized and compared with available experimental data in Table 1. As already obtained by other authors 63, 64, the systematic PBE error in $E_{\text {coh }}$ is about $11 \%$ for $\mathrm{Ti}, 6 \%$ for $\mathrm{Nb}$, and less than $1 \%$ for the rest. However, since the experimental ordering in $E_{\text {coh }}$ is still correctly reproduced, possible errors in the mixing of the elements within the alloys can be disregarded. The calculated bulk, shear, and Young's moduli are in a good 
Table 1: Equilibrium lattice constants $a(\AA)$ and $c(\AA)$, cohesive energy $E_{\text {coh }}$ (eV/atom), bulk modulus $B(\mathrm{GPa})$, shear modulus $G(\mathrm{GPa})$, Young's modulus $E$ (GPa), Poisson ratio $\nu$, Zener anisotropy $A_{Z}$, and Voight-Reuss elastic anisotropy $A_{G_{V R}}$ for the single-element metals. Experimental values for lattice constants [47, cohesive energies 68, and elastic moduli [65] are given in parentheses.

\begin{tabular}{lcccccccc}
\hline Metal & $a, c$ & $E_{\text {coh }}$ & $B$ & $G$ & $E$ & $\nu$ & $A_{Z}$ & $A_{G_{V R}}$ \\
\cline { 1 - 7 } Ti (hcp) & $2.93,4.66$ & -5.4 & 112.1 & 46 & 121.4 & 0.32 & 0.87 & 0.002 \\
& $(2.95,4.69)$ & $(-4.85)$ & $(108.4)$ & $(45.6)$ & $(120.2)$ & - & - & - \\
Zr (hcp) & $3.24,5.17$ & -6.3 & 93.6 & 33.7 & 90.3 & 0.34 & 0.72 & 0.013 \\
& $(3.23,5.15)$ & $(-6.25)$ & $(89.8)$ & $(36.5)$ & $(97.1)$ & - & - & - \\
Nb (bcc) & 3.31 & -7.1 & 171.3 & 29.3 & 83.2 & 0.42 & 0.32 & 0.150 \\
& $(3.30)$ & $(-7.57)$ & $(170.3)$ & $(37.5)$ & $(104.9)$ & - & - & - \\
Ta (bcc) & 3.32 & -8.1 & 196.1 & 65.7 & 177.2 & 0.35 & 1.44 & 0.016 \\
& $(3.31)$ & $(-8.10)$ & $(196.3)$ & $(69.2)$ & $(185.7)$ & - & - & - \\
Mo (bcc) & 3.16 & -6.6 & 260 & 122.3 & 317.2 & 0.30 & 0.67 & 0.019 \\
& $(3.15)$ & $(-6.82)$ & $(261.2)$ & $(125.6)$ & $(324.8)$ & - & - & - \\
\cline { 1 - 5 } & & & & & & & &
\end{tabular}

agreement with the experimental values [65. Moreover, the anisotropy ratio $\left(A_{G_{V R}}\right)$ is very low for most of the elements, which indicates the validity of the calculated results. The only exception is $\mathrm{Nb}$, for which the theoretical $G$ and $E$ are about $20 \%$ smaller than the experimental ones, which is understood given that the anisotropy ratios, $A_{G_{V R}}$ and $A_{Z}$, are relatively far from the correct limits. As previously noted by other authors [66, 67, this problem appears because present DFT calculations tend to highly underestimate the elastic constant $C_{44}$ as compared to the experimental value for $\mathrm{Nb}$.

We have analyzed five alloys in terms of structure and elastic properties in this study, namely, $\mathrm{Ti}_{2} \mathrm{Zr}_{2} \mathrm{Nb}_{2} \mathrm{Ta}_{2}, \mathrm{Ti}_{n} \mathrm{Zr}_{n} \mathrm{Nb}_{n} \mathrm{Ta}_{n} \mathrm{Mo}_{n}(n=2,4), \mathrm{Ti}_{2} \mathrm{Zr}_{1} \mathrm{Nb}_{2} \mathrm{Ta}_{3} \mathrm{Mo}_{2}$, and $\mathrm{Ti}_{3} \mathrm{Zr}_{4} \mathrm{Nb}_{1} \mathrm{Ta}_{1} \mathrm{Mo}_{1}$. In all these cases, the crystal-structure search procedure has been the following. The evolutionary algorithm produces 20 different structures in each generation (except for the $\mathrm{Ti}_{4} \mathrm{Zr}_{4} \mathrm{Nb}_{4} \mathrm{Ta}_{4} \mathrm{Mo}_{4}$, for which this number was 30) that are next relaxed by spin-polarized DFT until the forces 
on the ions are less than $0.001 \mathrm{eV} / \AA^{3}$. In order to reduce the computational cost of such calculations, the relaxation for each structure is performed in five steps with increasing accuracy. In the first two steps, the atoms and cell shape are relaxed at constant volume using an energy cutoff in the plane wave basis set of $220 \mathrm{eV}$ and $293 \mathrm{eV}$, with low and normal precision, respectively. In the next two steps, the volume and shape of the structures from the second step are fully relaxed using cutoff energies of 320 and $400 \mathrm{eV}$. The final step is an accurate single-point calculation with no change in shape and volume of the cell. The reciprocal-space resolution for $\mathbf{k}$-points generation is set to $0.16,0.12,0.10$, 0.08 , and 0.06 in units of the modulus of the reciprocal lattice vectors in each relaxation step, so that the number of $\mathbf{k}$-points increases at each step. In the present study, the evolutionary algorithm was selected to stop when the best crystal structure (the one with the lowest free energy in the current generation) remains the same during 25 generations.

Figure 1 shows the energy per atom for each of the structures produced in each generation, for four of the studied alloys. This figure serves to illustrate the diversity of the structures generated by the evolutionary algorithm. Note that in each generation the energies of the tested structures are widely spread. The reason is that there is always a small number of structures that are produced randomly in order to include new structural patterns into each generation. However, the lowest energy points in each plot of Fig. 1 converge to lower energies through generations. The mean energy and standard deviation that are also plotted for each generation provide information on the evolution and efficiency of the structure searching. In particular, the evolutionary algorithm stopped after 40 generations for $\mathrm{Ti}_{2} \mathrm{Zr}_{2} \mathrm{Nb}_{2} \mathrm{Ta}_{2}, 34$ generations for $\mathrm{Ti}_{2} \mathrm{Zr}_{2} \mathrm{Nb}_{2} \mathrm{Ta}_{2} \mathrm{Mo}_{2}$, and after 50 and 25 generations for the $\mathrm{Ti}_{2} \mathrm{Zr}_{1} \mathrm{Nb}_{2} \mathrm{Ta}_{3} \mathrm{Mo}_{2}$ and $\mathrm{Ti}_{3} \mathrm{Zr}_{4} \mathrm{Nb}_{1} \mathrm{Ta}_{1} \mathrm{Mo}_{1}$ alloys, respectively. 

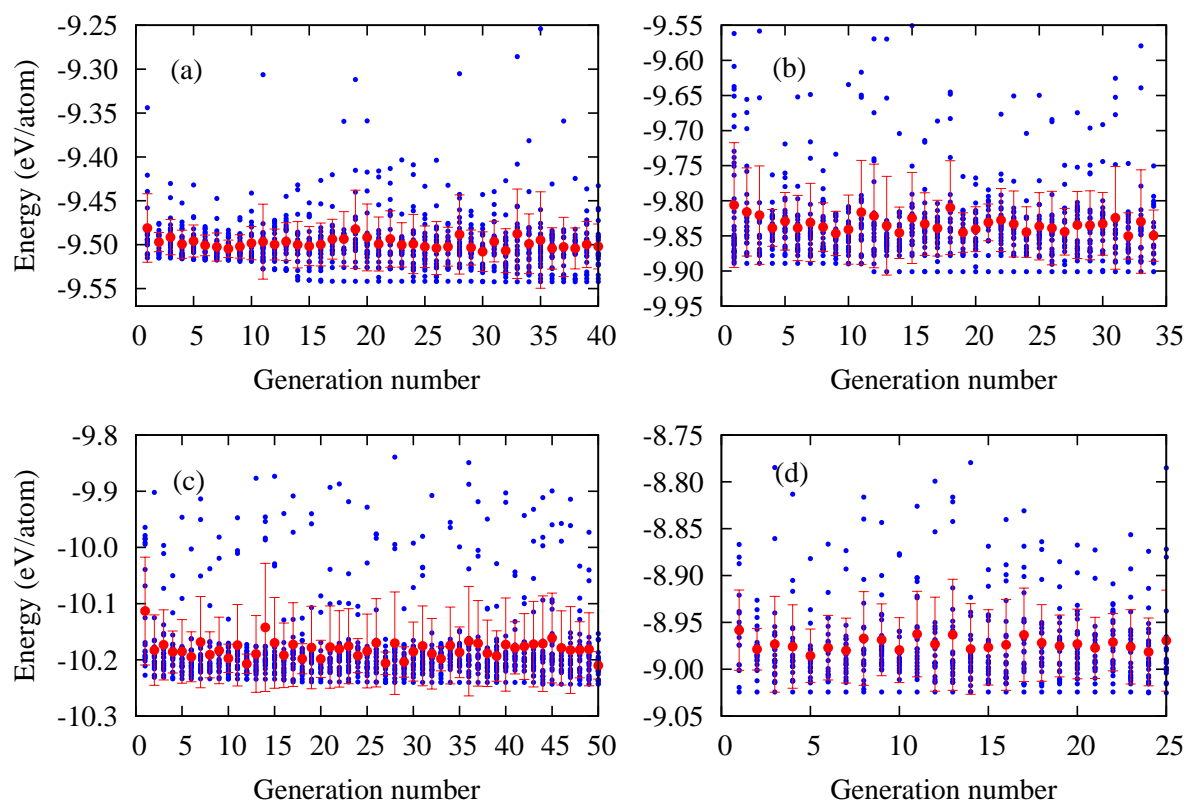

Figure 1: Energy per atom in $\mathrm{eV}$ for each of the structures produced at different generations by the USPEX calculation (blue dots). Each panel corresponds to a different alloy: (a) $\mathrm{Ti}_{2} \mathrm{Zr}_{2} \mathrm{Nb}_{2} \mathrm{Ta}_{2}$, (b) $\mathrm{Ti}_{2} \mathrm{Zr}_{2} \mathrm{Nb}_{2} \mathrm{Ta}_{2} \mathrm{Mo}_{2}$, (c) $\mathrm{Ti}_{2} \mathrm{Zr}_{1} \mathrm{Nb}_{2} \mathrm{Ta}_{3} \mathrm{Mo}_{2}$, and (d) $\mathrm{Ti}_{3} \mathrm{Zr}_{4} \mathrm{Nb}_{1} \mathrm{Ta}_{1} \mathrm{Mo}_{1}$. The average energy per generation as well as the corresponding standard deviation are plotted by full red circles and error bars, respectively.

\section{Results and discussion}

\subsection{Cell size effects}

In searching the crystal structure from first-principles, the use of small unit cells can considerably improve the computational performance, however one should first verify that reducing the unit cell size is not significantly altering the calculated properties. Therefore, as a first step in our study, we use the equiatomic $\mathrm{Ti}_{n} \mathrm{Zr}_{n} \mathrm{Nb}_{n} \mathrm{Ta}_{n} \mathrm{Mo}_{n}$ alloy as an example to check if the properties of interest are affected by the change in the number of atoms per species from $n=2$ to $n=8$, i.e., when the total number of atoms in the cell varies from 10 to 40. Importantly, it will be shown in Sec. 3.3 below that the experimental elastic properties are well reproduced by our calculations, what further supports the reliability of the predicted crystal structures. 
(a)

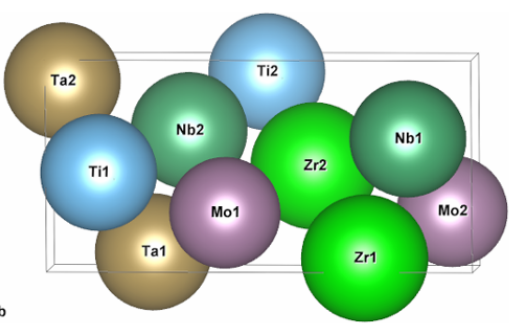

(b)

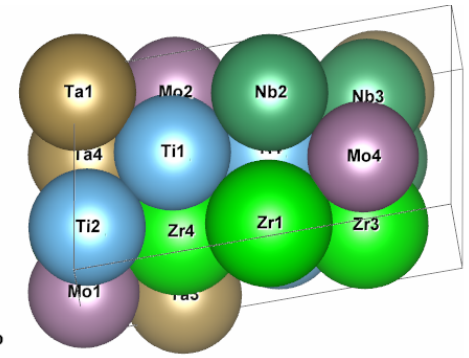

Figure 2: Predicted bcc structures for (a) $\mathrm{Ti}_{2} \mathrm{Zr}_{2} \mathrm{Nb}_{2} \mathrm{Ta}_{2} \mathrm{Mo}_{2}$ and (b) $\mathrm{Ti}_{4} \mathrm{Zr}_{4} \mathrm{Nb}_{4} \mathrm{Ta}_{4} \mathrm{Mo}_{4}$ Image produced by VESTA [69].

The stable structures predicted by the evolutionary algorithm combined with DFT for $\mathrm{Ti}_{2} \mathrm{Zr}_{2} \mathrm{Nb}_{2} \mathrm{Ta}_{2} \mathrm{Mo}_{2}$ and $\mathrm{Ti}_{4} \mathrm{Zr}_{4} \mathrm{Nb}_{4} \mathrm{Ta}_{4} \mathrm{Mo}_{4}$ are shown in Fig. 2 , Independently of the cell size, the obtained crystal structures are bcc-like for all the $\mathrm{Ti}_{n} \mathrm{Zr}_{n} \mathrm{Nb}_{n} \mathrm{Ta}_{n} \mathrm{Mo}_{n}$ alloys, in agreement with the experimental observations. However, the atomic positions are different in cells of different sizes. Therefore, we also calculate the elastic moduli in order to verify how the elastic properties are affected by the different arrangement of the atoms in the lattice. In these calculations the $\mathbf{k}$-points grid is chosen according to the symmetry and size of each unit cell, namely, $12 \times 4 \times 8$ for $\mathrm{Ti}_{2} \mathrm{Zr}_{2} \mathrm{Nb}_{2} \mathrm{Ta}_{2} \mathrm{Mo}_{2}, 6 \times 4 \times 8$ for $\mathrm{Ti}_{4} \mathrm{Zr}_{4} \mathrm{Nb}_{4} \mathrm{Ta}_{4} \mathrm{Mo}_{4}$, and $6 \times 4 \times 4$ for $\mathrm{Ti}_{6} \mathrm{Zr}_{6} \mathrm{Nb}_{6} \mathrm{Ta}_{6} \mathrm{Mo}_{6}$ and $\mathrm{Ti}_{8} \mathrm{Zr}_{8} \mathrm{Nb}_{8} \mathrm{Ta}_{8} \mathrm{Mo}_{8}$. All the alloys satisfy the mechanical stability constraints on the elastic constants, i.e., $\mathrm{C}_{11}>\mathrm{C}_{12}$, $\mathrm{C}_{11}+2 \mathrm{C}_{12}>0$, and $\mathrm{C}_{44}>0$ [49]. As Table 2 shows, the lattice parameter [70] and cohesive energy are very close for all the unit cell sizes. The differences in the bulk modulus $B$ and the Poisson ratio $\nu$ are minor. Slightly larger differences are found in the shear $G$ and Young's $E$ moduli, but the largest difference in $E$, which is obtained between the cells with 10 and 30 atoms, is not larger than $15 \%$. The deviations from 1 for $A_{Z}$ and from 0 for $A_{G_{V R}}$ anisotropy ratios are small for all the unit cell sizes and thus all the structures can be considered isotropic.

As an additional test, we also calculate for the $\mathrm{Ti}_{4} \mathrm{Zr}_{4} \mathrm{Nb}_{4} \mathrm{Ta}_{4} \mathrm{Mo}_{4}$ alloy the elastic moduli of its corresponding quasi-random structure. The latter is ob- 
Table 2: Calculated lattice constant $a(\AA)$, cohesive energy $E_{\text {coh }}(\mathrm{eV} /$ atom), bulk modulus $B(\mathrm{GPa})$, shear modulus $G(\mathrm{GPa})$, Young's modulus $E(\mathrm{GPa})$, Poisson ratio $\nu$, Pugh ratio $B / G$, Zener anisotropy $A_{Z}$, and Voight-Reuss elastic anisotropy $A_{G_{V R}}$ for the $\mathrm{Ti}_{n} \mathrm{Zr}_{n} \mathrm{Nb}_{n} \mathrm{Ta}_{n} \mathrm{Mo}_{n}(n=2-8)$ alloy structures and the quasi-random structure (QRS) obtained from the $\mathrm{Ti}_{4} \mathrm{Zr}_{4} \mathrm{Nb}_{4} \mathrm{Ta}_{4} \mathrm{Mo}_{4}$ alloy. The results for the $\mathrm{Ti}_{2} \mathrm{Zr}_{2} \mathrm{Nb}_{2} \mathrm{Ta}_{2}$ alloy are shown as well.

\begin{tabular}{|c|c|c|c|c|c|c|c|c|c|}
\hline Alloy & $a$ & $E_{\mathrm{coh}}$ & $B$ & $G$ & $E$ & $\nu$ & $B / G$ & $A_{Z}$ & $A_{G_{V R}}$ \\
\hline $\mathrm{Ti}_{2} \mathrm{Zr}_{2} \mathrm{Nb}_{2} \mathrm{Ta}_{2} \mathrm{Mo}_{2}$ & $3.32 \pm 0.19$ & -6.75 & 147.7 & 53.8 & 144.0 & 0.34 & 2.74 & 0.83 & 0.0043 \\
\hline $\mathrm{Ti}_{4} \mathrm{Zr}_{4} \mathrm{Nb}_{4} \mathrm{Ta}_{4} \mathrm{Mo}_{4}$ & $3.30 \pm 0.12$ & -6.73 & 148.0 & 52.0 & 139.7 & 0.34 & 2.85 & 1.04 & 0.0002 \\
\hline $\mathrm{Ti}_{6} \mathrm{Zr}_{6} \mathrm{Nb}_{6} \mathrm{Ta}_{6} \mathrm{Mo}_{6}$ & $3.30 \pm 0.12$ & -6.72 & 148.8 & 44.9 & 122.3 & 0.36 & 3.32 & 1.12 & 0.0010 \\
\hline $\mathrm{Ti}_{8} \mathrm{Zr}_{8} \mathrm{Nb}_{8} \mathrm{Ta}_{8} \mathrm{Mo}_{8}$ & $3.31 \pm 0.12$ & -6.72 & 148.0 & 47.7 & 129.3 & 0.35 & 3.10 & 1.04 & 0.0200 \\
\hline $\mathrm{Ti}_{4} \mathrm{Zr}_{4} \mathrm{Nb}_{4} \mathrm{Ta}_{4} \mathrm{Mo}_{4}$ (QRS) & $3.30 \pm 0.12$ & -6.72 & 147.0 & 50.0 & 135.5 & 0.34 & 2.92 & 1.04 & 0.0002 \\
\hline $\mathrm{Ti}_{2} \mathrm{Zr}_{2} \mathrm{Nb}_{2} \mathrm{Ta}_{2}$ & $3.50 \pm 0.28$ & -6.70 & 130.3 & 39.9 & 109.0 & 0.36 & 3.27 & 0.93 & 0.0006 \\
\hline
\end{tabular}

tained using the quasi-random structure technique implemented in the USPEX code, as mentioned in Sec. 2.1. Table 2 shows that the elastic moduli of this new structure and of the original structure are very similar. In view of these results and the fact that the differences in the elastic moduli are not significant among four different unit cell sizes, we continue the analysis for the rest of alloys using unit cells of 10 atoms, since this cell-size facilitates the computationally demanding calculations of the elastic tensor. In the following, we will refer to the structure and composition of the $\mathrm{Ti}_{2} \mathrm{Zr}_{2} \mathrm{Nb}_{2} \mathrm{Ta}_{2} \mathrm{Mo}_{2}$ alloy as the main phase.

Besides the best structure of $\mathrm{Ti}_{2} \mathrm{Zr}_{2} \mathrm{Nb}_{2} \mathrm{Ta}_{2} \mathrm{Mo}_{2}$, we have analyzed as well the list of the obtained 20 best crystal structures from all the generations. Many of them possess bcc lattices with the cohesive energy per atom varying within $[-6.66,-6.70] \mathrm{eV}$. The second best structure has the same configuration as the main phase [see Fig. 2(a)] but the $\mathrm{Nb}_{1}$ and $\mathrm{Ta}_{2}$ atoms are interchanged. The corresponding computed elastic constants and moduli for this structure appear to be almost identical to the ones of the $\mathrm{Ti}_{2} \mathrm{Zr}_{2} \mathrm{Nb}_{2} \mathrm{Ta}_{2} \mathrm{Mo}_{2}$ main phase. Such similarities suggest that the chemical composition and the type of lattice are more important for the elastic properties than the exact arrangement of the 
atoms in the lattice.

\subsection{The role of Mo in improving the mechanical properties}

As explained in Refs. 28, 27, the selection of the elements conforming the alloy requires the chemical biocompatibility of all the elements and superior mechanical properties. In this respect, the main reason to add Mo in the mixture was to improve the wear resistance in view of the high elastic modulus of this metal as compared to that of the other atomic constituents (see Table 11). We, therefore, find meaningful to analyze from first principles if this is actually the case by comparing the elastic properties of the equiatomic $(n=2)$ alloys with and without molybdenum.

The most stable structure predicted by the evolutionary algorithm for the $\mathrm{Ti}_{2} \mathrm{Zr}_{2} \mathrm{Nb}_{2} \mathrm{Ta}_{2}$ alloy is also bcc with a lattice parameter [70] $a=3.50 \AA( \pm 0.28 \AA)$. Comparing the lattice constants of the equiatomic $\mathrm{Ti}_{2} \mathrm{Zr}_{2} \mathrm{Nb}_{2} \mathrm{Ta}_{2} \mathrm{Mo}_{2}$ and $\mathrm{Ti}_{2} \mathrm{Zr}_{2} \mathrm{Nb}_{2} \mathrm{Ta}_{2}$ alloys (Table2), we observe that addition of Mo leads to the formation of a more compact structure in which the lattice constant $a$ is decreased in about $6 \%$. The same behavior is observed in the recent experimental work by Wang et al. [71, in which they study the (TiZrNbTa) $)_{100-x} \operatorname{Mo}_{x}(0 \leq x \leq 20)$ alloys. They also find that the lattice parameter is reduced with higher content of Mo. In spite of this reduction, we observe here that the theoretical cohesive energies are basically identical in both alloys. Our DFT results for the elastic moduli of $\mathrm{Ti}_{2} \mathrm{Zr}_{2} \mathrm{Nb}_{2} \mathrm{Ta}_{2}$ are shown in Table 2 As expected, the addition of Mo increases all the elastic moduli of the alloy. The Young's modulus we obtain for the $\mathrm{Ti}_{2} \mathrm{Zr}_{2} \mathrm{Nb}_{2} \mathrm{Ta}_{2} \mathrm{Mo}_{2}$ and $\mathrm{Ti}_{2} \mathrm{Zr}_{2} \mathrm{Nb}_{2} \mathrm{Ta}_{2}$ alloys are 144 and $109 \mathrm{GPa}$, which are in a good agreement with experimental values of $155 \pm 6$ and $115 \pm 3 \mathrm{GPa}$, respectively [71. Similar value of the elastic modulus for the TiZrNbTa alloy was obtained by Nguyen et. al. 26], which is $116 \pm 6 \mathrm{GPa}$, in agreement with our calculated result. It is interesting to note that for each alloy, the elastic moduli are slightly smaller than the arithmetic average calculated from the values of the pure elements. For example, the average bulk moduli are 166.6 and $143.3 \mathrm{GPa}$ for $\mathrm{Ti}_{2} \mathrm{Zr}_{2} \mathrm{Nb}_{2} \mathrm{Ta}_{2} \mathrm{Mo}_{2}$ and $\mathrm{Ti}_{2} \mathrm{Zr}_{2} \mathrm{Nb}_{2} \mathrm{Ta}_{2}$ alloys, respectively, while the actual values are 147.7 and 

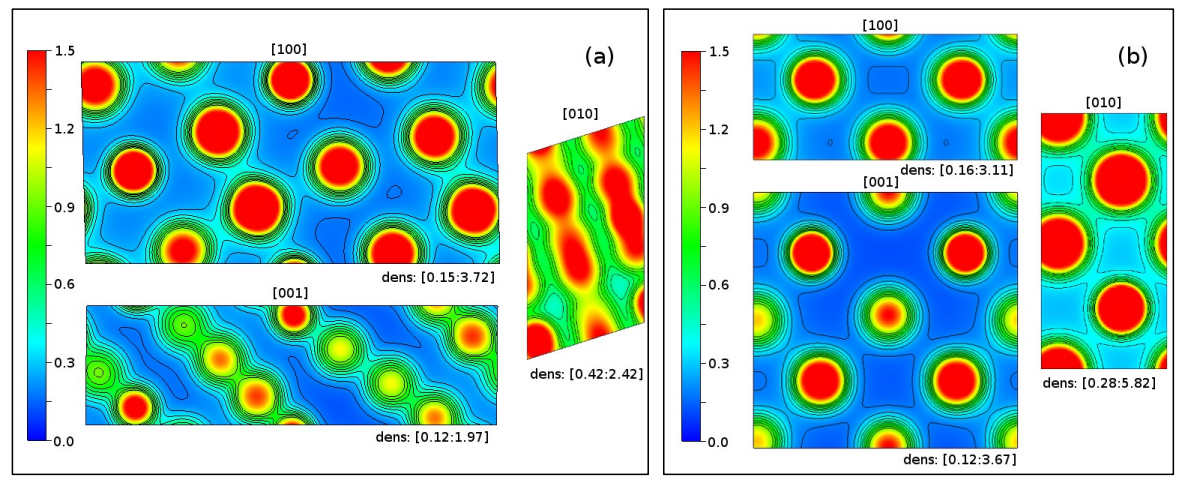

Figure 3: Projected charge density isosurfaces along [100], [010], and [001] crystallographic directions for (a) $\mathrm{Ti}_{2} \mathrm{Zr}_{2} \mathrm{Nb}_{2} \mathrm{Ta}_{2} \mathrm{Mo}_{2}$ and (b) $\mathrm{Ti}_{2} \mathrm{Zr}_{2} \mathrm{Nb}_{2} \mathrm{Ta}_{2}$. The color code shows the charge density in electrons $/ \AA^{3}$ and is limited to $[0: 1.5]$ in all the cases. Real limits are shown below each plot. Image produced by VESTA 69 .

130.3 GPa. It has been previously shown that the average method works well for other refractory MPEAs [72.

In order to better understand the effect of Mo on the elastic properties, we analyze and compare the electron density of the $\mathrm{Ti}_{2} \mathrm{Zr}_{2} \mathrm{Nb}_{2} \mathrm{Ta}_{2} \mathrm{Mo}_{2}$ and $\mathrm{Ti}_{2} \mathrm{Zr}_{2} \mathrm{Nb}_{2} \mathrm{Ta}_{2}$ alloys. In Fig. 3 , the three color plots for each alloy show the isosurfaces of the electron density projected along the [100], [010], and [001] crystallographic directions. Each projection is calculated as the sum of a series of $(h k l)$ slices of the electron density along the $[h k l]$ direction. In order to allow for a quantitative comparison the projections in each alloy are done using the same vector length. A general observation is that the electron density in the interatomic region is smaller in the alloy without Mo, which actually has a smaller nominal electron density. As pointed above, the alloy containing Mo has a smaller lattice constant, i.e., a more compact structure that suggests stronger bonding between atoms in this alloy as compared to the $\mathrm{Ti}_{2} \mathrm{Zr}_{2} \mathrm{Nb}_{2} \mathrm{Ta}_{2}$ one. Note for instance the large region of low density that exists in the projection along the [001] direction for $\mathrm{Ti}_{2} \mathrm{Zr}_{2} \mathrm{Nb}_{2} \mathrm{Ta}_{2}$. The overall stronger bonds and higher interatomic density can explain the higher values of bulk, shear, and Young's moduli for the $\mathrm{Ti}_{2} \mathrm{Zr}_{2} \mathrm{Nb}_{2} \mathrm{Ta}_{2} \mathrm{Mo}_{2}$ alloy. 
In the experimental studies of Refs. 28, 27, the TiZrNbTaMo alloy was fabricated by arc melting equiatomic fractions of highly pure Ti, Zr, Nb, Ta, and Mo bulk materials. Using energy dispersive X-ray spectroscopy (EDX), the final average chemical composition of the formed alloy was determined to be $\operatorname{Ti}(19 \%) \operatorname{Zr}(21 \%) \operatorname{Nb}(19 \%) \operatorname{Ta}(21 \%) \mathrm{Mo}(20 \%)$ with an estimated error of $\pm 1 \%$ in the atomic percentage. The corresponding VEC value of 4.8 would predict the crystallization of the equiatomic alloy in the bcc structure as it was actually confirmed by X-ray diffraction (XRD). However, a more in-depth microcharacterization of the as-cast alloy assisted by scanning electron microscopy (SEM) revealed the coexistence of two distinct bcc phases. The predominant major phase (70\% of the alloy) with a lattice parameter $a=3.3 \AA$ was $\mathrm{Ti}_{15} \mathrm{Zr}_{10} \mathrm{Nb}_{20} \mathrm{Ta}_{31} \mathrm{Mo}_{24}$ $(\mathrm{VEC}=4.7)$ and the less abundant minor phase (30\% of the alloy) with $a=3.4 \AA$ was $\mathrm{Ti}_{24} \mathrm{Zr}_{43} \mathrm{Nb}_{12} \mathrm{Ta}_{8} \mathrm{Mo}_{13}(\mathrm{VEC}=4.4)$.

In our case, we approximate the major phase as $\mathrm{Ti}_{2} \mathrm{Zr}_{1} \mathrm{Nb}_{2} \mathrm{Ta}_{3} \mathrm{Mo}_{2}$ (VEC $=4.9)$ and the minor phase as $\mathrm{Ti}_{3} \mathrm{Zr}_{4} \mathrm{Nb}_{1} \mathrm{Ta}_{1} \mathrm{Mo}_{1}(\mathrm{VEC}=4.4)$. Figure 4 shows the structures predicted by the USPEX calculations for the major and minor phases of the alloy. The two phases possess a bcc crystal structure, which is consistent with the experimental observations and the VEC criterion. Remarkably, our calculated lattice parameters [70] are also in good agreement with the ones reported in experiments (see Table 3 ).

We have computed the elastic moduli for both major and minor phases, using a k-point mesh in the calculations of the elastic tensor of $6 \times 6 \times 12$ and $6 \times 8 \times 8$, respectively. The results are shown in Table 3 , together with the experimental values of $E[28$. The latter were obtained by applying the Oliver and Pharr method [73] to nanoindentation measurements performed on each phase. Even though our predicted Young's moduli are lower than the experimental values, our theoretical results exhibit the same trend. More precisely, $E$ of the major phase exceeds in about $30 \mathrm{GPa}$ the value of the minor phase. This behavior, i.e., the larger Young's modulus of the major phase as compared to that of the 

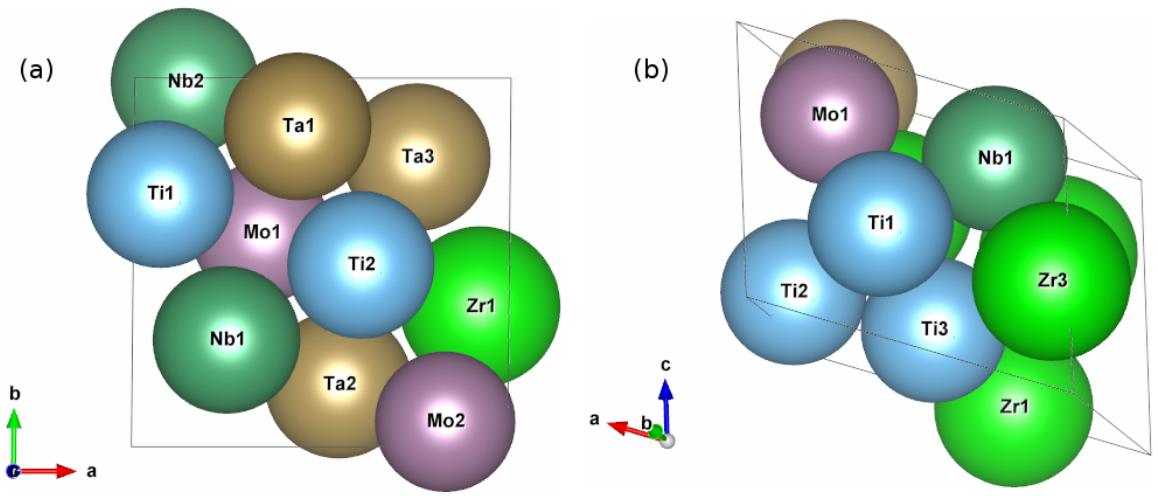

Figure 4: Predicted bcc structures for (a) the major phase $\mathrm{Ti}_{2} \mathrm{Zr}_{1} \mathrm{Nb}_{2} \mathrm{Ta}_{3} \mathrm{Mo}_{2}$ and (b) the minor phase $\mathrm{Ti}_{3} \mathrm{Zr}_{4} \mathrm{Nb}_{1} \mathrm{Ta}_{1} \mathrm{Mo}_{1}$. Image produced by vesta 69 .

Table 3: Calculated lattice constant $a(\AA)$, cohesive energy $E_{\text {coh }}(\mathrm{eV} /$ atom), bulk modulus $B(\mathrm{GPa})$, shear modulus $G(\mathrm{GPa})$, Young's modulus $E(\mathrm{GPa})$, Poisson ratio $\nu$, and Pugh ratio $B / G$, for the major $\left(\mathrm{Ti}_{2} \mathrm{Zr}_{1} \mathrm{Nb}_{2} \mathrm{Ta}_{3} \mathrm{Mo}_{2}\right)$ and minor $\left(\mathrm{Ti}_{3} \mathrm{Zr}_{4} \mathrm{Nb}_{1} \mathrm{Ta}_{1} \mathrm{Mo}_{1}\right)$ phases. The experimental values for $a$ and $E$ reported in Ref. [28] are indicated by the subscript 'exp'. The calculated anisotropy ratios $A_{Z}$ and $A_{G_{V R}}$ are shown as well.

\begin{tabular}{cccccccccccc}
\hline Phase & $a$ & $a_{\exp }$ & $E_{\text {coh }}$ & $B$ & $G$ & $E$ & $E_{\exp }$ & $\nu$ & $B / G$ & $A_{Z}$ & $A_{G_{V R}}$ \\
\cline { 1 - 5 } & $3.30 \pm 0.18$ & 3.31 & -6.94 & 163.4 & 53.1 & 143.6 & $161 \pm 7$ & 0.35 & 3.08 & 0.87 & 0.0023 \\
minor & $3.36 \pm 0.25$ & 3.38 & -6.32 & 115.3 & 41.3 & 110.7 & $133 \pm 6$ & 0.34 & 2.79 & 1.07 & 0.0005
\end{tabular}

minor phase, is due to the fact that the former phase has a higher content of stiff elements, such as Ta and Mo, than the latter.

Regarding the experimental value of the Young's modulus for the equiatomic TiZrNbTaMo, let us remark that the reported value of 153 GPa was determined as weighted by volume fractions of the two phases and thus is not measured directly [28]. Therefore, in order to correctly compare the results for the equiatomic phase, we calculate the average moduli as $E_{\mathrm{av}}=0.7 E_{\text {major }}+$ $0.3 E_{\text {minor }}$, where 0.7 and 0.3 are the volume fractions of the major and minor phases, respectively. Applying the same averaging-formula to the other elastic moduli, we obtain the following average bulk, shear, and Young's moduli, as well as Poisson's ratio: $B_{\mathrm{av}}=150.6 \mathrm{GPa}, G_{\mathrm{av}}=48.1 \mathrm{GPa}, E_{\mathrm{av}}=130.45 \mathrm{GPa}$, 
and $\nu_{\mathrm{av}}=0.33$. Similarly to the case of major and minor phases, the average Young's modulus is approximately $23 \mathrm{GPa}$ below the experimental value.

Experimentally, the Vickers microhardness $H_{V}$ is the usual macroscopic property that can be measured directly and serves to characterize the hardness of a material. The measured $H_{V}$ of the as-cast TiZrNbTaMo and TiZrNbTa alloys are $4.9 \pm 0.1$ and $3.2 \pm 0.1 \mathrm{GPa}$, respectively [28, 71]. In addition, Wang et al. 28] estimated from the nanoindentation tests that the values for the major and minor phases were $H_{V}=6.4$ and $5.7 \mathrm{GPa}$, respectively (for details please refer to Ref. [28]). There have been proposed various expressions for the calculation of the Vickers microhardness of an isotropic crystalline phase from the elastic moduli. For instance, Qiu et al. [74] use the following expression in terms of the Young's modulus and Poisson's ratio, $H_{V}^{\mathrm{Qiu}}=\frac{(1-2 \nu) E}{6(1+\nu)}$. Tian et al. [75] propose an empirical formula to estimate the microhardness from the bulk and shear moduli as $H_{V}^{\text {Tian }}=0.92(G / B)^{1.137} G^{0.708}$. Our results for the Vickers microhardness calculated with both methods are compared to the experimental $H_{V}$ in Table 4. For the equiatomic phases, the calculated $H_{V}$ are in a good agreement with the experimental values, although are slightly overestimated when using the Qiu et al. method. On the contrary, the values of the calculated microhardness for the major and minor phases are lower than the values from the experimental reference. However, as already mentioned in Ref. [28], their estimated experimental values for the major and minor phases are approximately $31 \%$ and $16 \%$ higher than the directly measured microhardness for the overall specimen due to the indentation size effect.

The so-called Pugh ratio $B / G$ is often used as an indicator of the ductilebrittle behavior of the materials [76] and has also been applied to MPEAs [77. Ductile materials usually have high values of $B / G(>1.75)$, while brittle materials have low values of $B / G(<1.75)$. The Poisson's ratio $\nu$ can also be used to predict the ductile-brittle behavior [78. It has been shown that bulk metallic glasses with $\nu>0.31$ are ductile and that this parameter can also be applied to MPEAs. The results in Tables 2 and 3 show that all the phases have very high values of $B / G$ and Poisson's ratio that are associated with ductility. Among the 
Table 4: Experimental $H_{V}^{\exp }$ and calculated $H_{V}^{\text {Qiu }}$ and $H_{V}^{\text {Tian }}$ Vickers microhardness for the TiZrNbTaMo main, major, and minor phases and TiZrNbTa. Experimental values from Refs. 28, 71.

\begin{tabular}{llll}
\hline Phase & $H_{V}^{\exp }(\mathrm{GPa})$ & $H_{V}^{\mathrm{Qiu}}(\mathrm{GPa})$ & $H_{V}^{\mathrm{Tian}}(\mathrm{GPa})$ \\
\hline main & $4.9 \pm 0.1$ & 5.7 & 4.9 \\
major & 6.4 & 5.3 & 4.3 \\
minor & 5.7 & 4.4 & 4.0 \\
TiZrNbTa & $3.2 \pm 0.1$ & 3.7 & 3.3 \\
\hline
\end{tabular}

three phases, the major phase is more ductile than the main and minor phases. Similar conclusions can be drawn from the analysis of the Cauchy pressure, which is defined from the elastic constants as $P_{C}=\left(C_{12}-C_{44}\right)$ (we use average values of $C_{i j}$ here). The positive values of the Cauchy pressure are associated with the metallic character of the bonding and with ductility [79, 80]. All of the alloys studied in this work have positive Cauchy pressures. The highest value of $75 \mathrm{GPa}$ corresponds to the major phase, while the values of $46 \mathrm{GPa}$ and 58 GPa correspond to the minor and main phases, respectively.

Figure 5 shows three-dimensional plots of the single-crystalline Young's modulus [49] along [hkl] crystallographic directions, calculated and plotted with the SC-EMA (Self-Consistent Calculations - Elasticity of Multi-phase Aggregates) software [81, 82, 83, 84] using our computed elastic constants. The values on both the color scale and the axes are Young's modulus in GPa. Note that the scale is different for each phase. One can observe that for the main and major phases the Young's moduli have a rather strong dependence on the orientation. The maximum value of $176 \mathrm{GPa}$ for the main phase is along the [100] and [100] directions, while a minimum value of $118 \mathrm{GPa}$ is along the [011] (upper red area) and [011] (lower red area) directions [Fig. 5(a)]. The picture is somewhat reversed for the major phase [Fig. 5(b)]. Thus, these two systems have a certain degree of anisotropy. In contrast, the shape of the characteristic surface of Young's modulus for the minor phase is almost spherical [Fig. 5(c)], which indicates that this phase is more isotropic than the main and major phases. This 

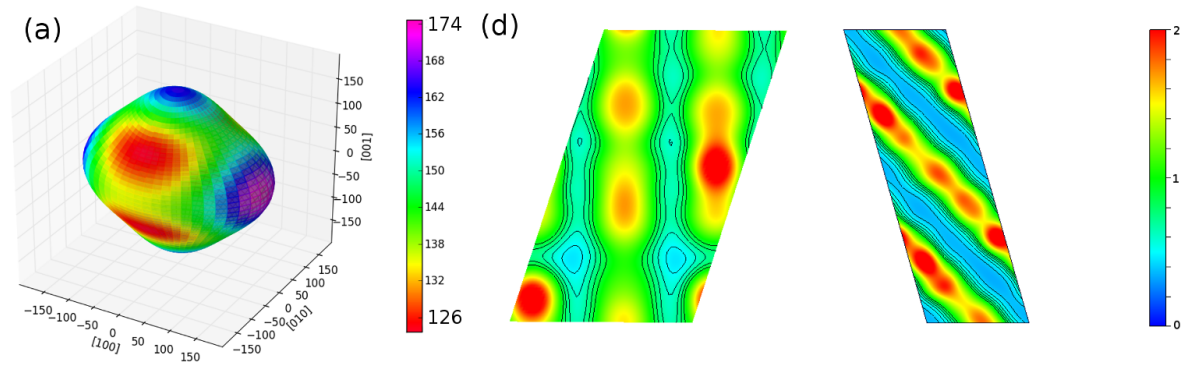

(b)
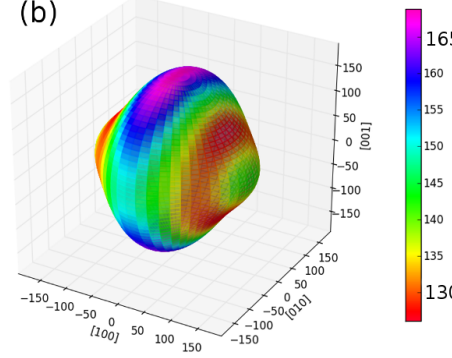

(e)
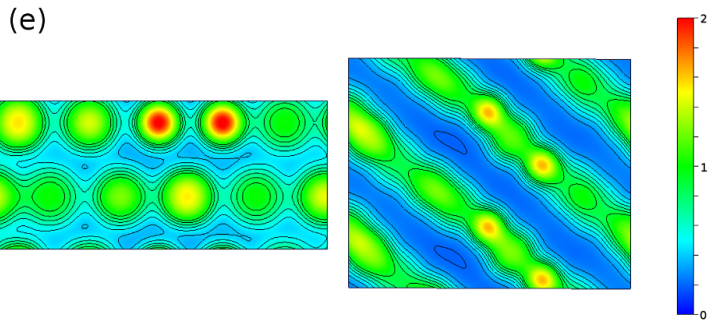

(c)

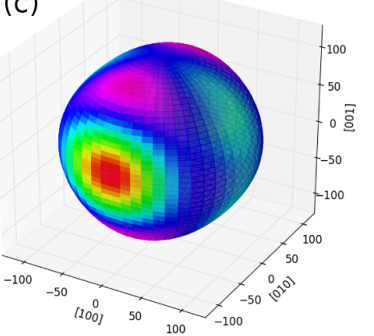

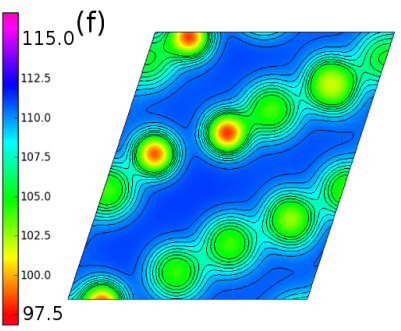

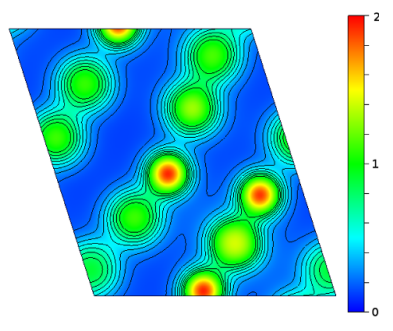

Figure 5: Left panels: direction dependent Young's modulus for (a) the main phase $\mathrm{Ti}_{2} \mathrm{Zr}_{2} \mathrm{Nb}_{2} \mathrm{Ta}_{2} \mathrm{Mo}_{2}$, (b) the major phase $\mathrm{Ti}_{2} \mathrm{Zr}_{1} \mathrm{Nb}_{2} \mathrm{Ta}_{3} \mathrm{Mo}_{2}$, and (c) the minor phase $\mathrm{Ti}_{3} \mathrm{Zr}_{4} \mathrm{Nb}_{1} \mathrm{Ta}_{1} \mathrm{Mo}_{1}$. Color scale and axes values are in GPa. Visualized by SC-EMA code $81,82,83,84$. Middle and right panels represent the charge density isosurfaces projected along the directions of maximum and minimum Young's moduli, respectively, for (d) main, (e) major, and (f) minor phases. The contour lines have an interval of 0.09 electrons $/ \AA^{3}$. Images produced by VESTA [69]. See text for more details. 
is consistent with the values of $A_{Z}$ and $A_{G_{V R}}$ for the three phases presented in Tables 2 and 3 , which also show that the minor phase is the most isotropic of the three phases.

In order to gain insight into the dependence of the Young's modulus on the crystallographic direction, we analyze the atomic bonding character in the three alloys. The projected charge density isosurfaces along the direction of maximum and minimum of the Young's modulus for the main, major, and minor phases are shown in Figs. 5(d)-(f). As explained above, each projection is calculated as the sum of various $(h k l)$ slices of the electron density along the $[h k l]$ direction. Larger values of the Young's modulus indicate that the material is stiffer. Thus, we expect stronger bonding in the direction of the maximum Young's modulus. For the main phase, we show in Fig. 5(d) the isosurfaces of the charge density projected along the [100] direction on the left and along the [011] direction on the right, i.e., the directions along which the Young's modulus takes the maximum and minimum values, respectively. In general, the overall projection of the charge density along [100] is larger than along [011], where extreme regions of high and low density appear in alternate stripes. The same is observed in the case of the major phase [Fig. 5(e)]. Remarkably, almost no difference between the two charge density projections is observed in the case of the minor phase [Fig. [5(f)], for which the Young's modulus is much more isotropic.

As a final remark in this section, we note that there is a direct correlation between the VEC and the elastic modulus. The phase with higher VEC has higher bulk modulus. The same correlation was already observed for other refractory alloys 25]. This might be explained in terms of the electronic structure that will be discussed in the next subsection.

\subsection{Electronic properties of the TiZrNbTaMo alloy phases}

In order to understand better the obtained elastic properties of the studied alloys, we analyze the electron density of states (DOS) and the atom-resolved partial electron density of states (pDOS), both calculated using the VASP im-

plementation. In order to increase the DOS resolution the $\mathbf{k}$-point meshes have 
been increased to $18 \times 9 \times 12$ for the main phase, $12 \times 12 \times 18$ for the major phase, and $12 \times 18 \times 18$ for the minor phase.

Besides DOS we compute as well the so-called crystal orbital Hamilton populations (COHP) 85]. The COHP separates the DOS into bonding, nonbonding, and antibonding contributions. The calculations are done by using the LOBSTER (Local Orbital Basis Suite Towards Electronic-Structure Reconstruction) code [86, 87, 88, 89, which enables chemical-bonding analysis based on the VASP output. The underlying physical reasoning in doing this analysis is as follows. For each pair of neighboring atoms, the interaction between the $\phi_{\mu}$ and $\phi_{\nu}$ orbitals, which are respectively centered at each atom, is described by the Hamiltonian matrix element $H_{\mu \nu}=\left\langle\phi_{\mu}|\hat{H}| \phi_{\nu}\right\rangle$-the details of the transfer from plane waves to atomic orbitals can be found in Ref. [87]. The multiplication of the Hamiltonian matrix elements by the corresponding DOS matrix serves as a quantitative measure of the bond strength. The product either lowers (bonding) or raises (antibonding) the band-structure energy. Thus, energy-resolved $\mathrm{COHP}(\mathrm{E})$ plots allow distinguishing between bonding, nonbonding (no energetic effect), and antibonding contributions [87]. Usually, the -COHP values are plotted so that bonding is characterized by positive values and antibonding by negative values.

Figure 6(left panels) shows the DOS for the main, major, and minor phases. A deep minimum close to the Fermi level is observed in the three cases, although it is less pronounced in the minor phase. This characteristic minimum, appearing in alloys formed by $d$-elements, is usually called a pseudogap [90, 91]. The corresponding pDOS curves show that all the atoms almost equally contribute to the total DOS in the pseudogap region, with Ti having the largest value at $E_{\mathrm{F}}$. At positive energies, Mo and Ti make a major contribution to the total DOS, while Zr and Ta mainly contribute to the appearance of the peak around $5 \mathrm{eV}$. The presence of this pseudogap and, more precisely, the position of $E_{\mathrm{F}}$ with respect to the pseudogap is associated with the structural stability of the intermetallic compound. The pseudogap usually separates bonding states from antibonding (nonbonding) states. As shown in Fig. 6. $E_{\mathrm{F}}$ lies to the left of 

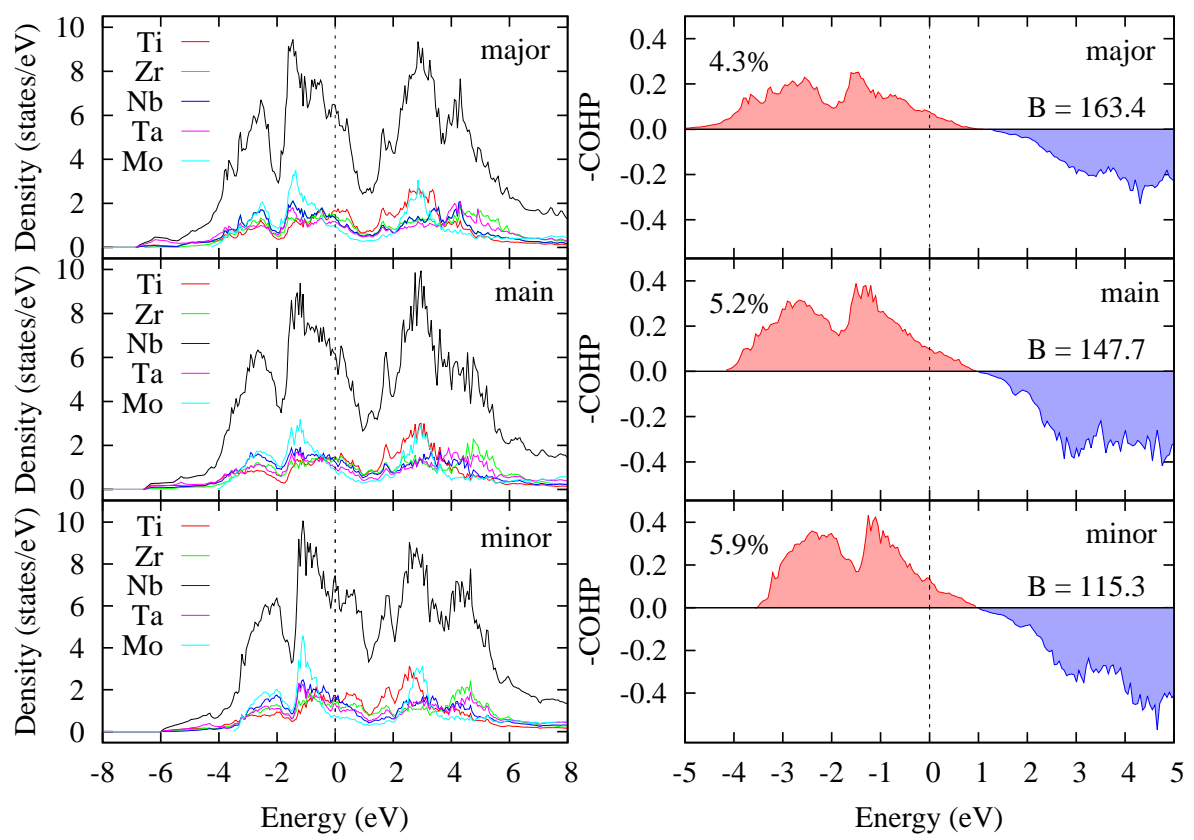

Figure 6: Left panels: total (black curves) and $\mathrm{Ti}, \mathrm{Zr}, \mathrm{Nb}$, Ta, Mo partial electronic density of states (colored curves). Right panels: Crystal orbital Hamilton population -COHP for bonding (red-shaded area) and antibonding (blue-shaded area). Results for the main phase $\mathrm{Ti}_{2} \mathrm{Zr}_{2} \mathrm{Nb}_{2} \mathrm{Ta}_{2} \mathrm{Mo}_{2}$ (upper panels), the major phase $\mathrm{Ti}_{2} \mathrm{Zr}_{1} \mathrm{Nb}_{2} \mathrm{Ta}_{3} \mathrm{Mo}_{2}$ (middle panels), and the minor phase $\mathrm{Ti}_{3} \mathrm{Zr}_{4} \mathrm{Nb}_{1} \mathrm{Ta}_{1} \mathrm{Mo}_{1}$ (bottom panels). In all plots the Fermi level is shown by a vertical dashed line. The percentage of the unoccupied part of the bonding area and the Bulk modulus (GPa) are indicated in the - COHP plots for each phase. 
the pseudogap, i.e., within the bonding states. The same conclusion is also extracted from the COHP results plotted in the right panels of Fig. 6. These results clearly show that not all the bonding states are completely filled. Thus, more electrons are needed to reach the maximum structural stability in these systems [91].

Although the COHP plots for all three systems look similar, one can notice that for the minor phase, the bonding region is shifted to the right with respect to the Fermi level, as compared to the main and major phases. Since the number of states between the different phases differs, we also compute the percentage of unoccupied bonding states in each case to facilitate the comparison. The values, which are written on each COHP plot, confirm that the bonding states population increases as going from minor to main and to major phases, following this order. Note that this ordering correlates well with the values of the cohesive energies per atom that we obtain for each phase (Tables 2 and 3 ) and, hence, with their corresponding stability. Furthermore, the occupation of the bonding states explains the values obtained for the bulk modulus and the Poisson's ratio. It has been shown that the full occupation of the bonding states results in high bulk modulus and low Poisson's ratio 92. As an example, for pure transition metals $\mathrm{Ti}(B=112 \mathrm{GPa}, \nu=0.32)$ and $\mathrm{Cr}(B=160 \mathrm{GPa}, \nu=0.21)$, the Fermi level falls within the bonding region in $\mathrm{Ti}$, and just between bonding and antibonding regions in $\mathrm{Cr}$ 93. The same behavior is observed for the three phases of the alloy we study here, i.e., $B$ increases as the percentage of unoccupied bonding states $\rho_{\mathrm{bond}}^{\mathrm{un}}$ decreases. Specifically, the minor phase has the lowest $B(115.3 \mathrm{GPa})$ and the highest $\rho_{\text {bond }}^{\text {un }}(5.9 \%)$, the main phase has the intermediate values of $147.7 \mathrm{GPa}$ and $5.2 \%$, and the major phase has the highest $B(163.4 \mathrm{GPa})$ and the lowest $\rho_{\mathrm{bond}}^{\mathrm{un}}(4.3 \%)$. The obtained differences in the $E$ and $G$ values among the three alloy phases correlate with the different occupation of the bonding states in a way similar to the bulk modulus B.

The detailed analysis of the COHP for each pair of atoms in the major and minor phases shows that among 45 atomic pairs only 18 contribute to the unoccupied bonding states in the major phase against 26 pairs in the minor 
phase. These contributions mostly come from the Ti-Ta, Ti-Zr, Nb-Ta, and Ta-Ta bonding in the major phase. In the minor phase the unoccupied bonding states are observed in the COHPs of the different Ti-Ti, Ti-Zr, and $\mathrm{Zr}-\mathrm{Zr}$ atomic pairs. This explains the larger percentage of the unoccupied bonding states in the total COHP of the minor phase, with a high content of $\mathrm{Ti}$ and $\mathrm{Zr}$, as compared to the major phase.

\section{Conclusions}

We have performed an ab initio study of the structure and properties of the experimentally investigated TiZrNbTaMo multi-principal element alloy that has a potential to be used as a bone implant material. We have applied an evolutionary algorithm in combination with DFT calculations, which correctly predicted the experimentally observed bcc structures for all the alloy phases. By comparing the results for different sizes of the unit cell, we have shown that it is not necessary to use a large unit cell in order to properly describe the elastic properties of the complex alloy. The particular chemical ordering of atoms and the unit cell size seem to be less important than the chemical composition for the elastic properties of the TiZrNbTaMo multi-principal element alloy, as our results show.

The DFT-based calculations of the elastic properties qualitatively agree with the experiment, i.e., correctly predict which of the phases has lower and higher values of the elastic moduli. All the studied alloy phases have large values of the Poisson's ratio and the Pugh ratio, indicating their high ductility. The detailed analysis of the direction-dependent Young's modulus, as well as the calculated anisotropy ratios, have shown that the minor phase is the most isotropic.

The analysis of the electronic density of states as well as the bondingantibonding character (COHP) of the different TiZrNbTaMo alloy phases show the presence of unoccupied bonding states. The different percentage of the energy-weighted unoccupied bonding states explains the differences in the elastic moduli of the three alloy phases. In particular, the values of the elastic 
moduli are higher for the phase with a higher percentage of (energy-weighted) occupied bonding states.

\section{Declaration of interest}

None.

\section{Acknowledgements}

We are grateful to Mgr. Martin Friák for his assistance in using the SCEMA code. We thank Dr. Peter Koval for helpful comments and discussion on the manuscript. The work was partially financed by Gobierno Vasco through the ELKARTEK Project KK-2017/00007 and Spanish Ministerio de Economía, Industria y Competitividad [grant number FIS2016-76471-P]. Computational resources were provided by the DIPC and CFM/MPC computing centers.

\section{References}

[1] P.-K. Huang, J.-W. Yeh, T.-T. Shun, S.-K. Chen, Multi-principalelement alloys with improved oxidation and wear resistance for thermal spray coating, Adv. Eng. Mater. 6 (12) (2004) 74-78. arXiv:https: //onlinelibrary.wiley.com/doi/pdf/10.1002/adem.200300507, doi:10.1002/adem.200300507.

URL https://onlinelibrary.wiley.com/doi/abs/10.1002/adem. 200300507

[2] D. Miracle, O. Senkov, A critical review of high entropy alloys and related concepts, Acta Mater. 122 (2017) 488-511. doi:10.1016/j.actamat.2016.08.081.

URL http://www.sciencedirect.com/science/article/pii/ S1359645416306759 
[3] M. C. Gao, J.-W. Yeh, P. K. Liaw, Y. Zhang, High-Entropy Alloys, Springer, 2016. doi:10.1007/978-3-319-27013-5

URL https://wWw . springer.com/gp/book/9783319270111

[4] J.-W. Yeh, S.-K. Chen, S.-J. Lin, J.-Y. Gan, T.-S. Chin, T.-T. Shun, C.H. Tsau, S.-Y. Chang, Nanostructured high-entropy alloys with multiple principal elements: Novel alloy design concepts and outcomes, Adv. Eng. Mater. 6 (5) (2004) 299-303. doi:10.1002/adem. 200300567.

URL http://dx.doi.org/10.1002/adem.200300567

[5] B. Cantor, I. Chang, P. Knight, A. Vincent, Microstructural development in equiatomic multicomponent alloys, Mater. Sci. Eng. A 375 - 377 (2004) 213 - 218. doi:10.1016/j.msea.2003.10.257

URL http://www.sciencedirect.com/science/article/pii/ S0921509303009936

[6] B. Cantor, High-entropy alloys, in: K. J. Buschow, R. W. Cahn, M. C. Flemings, B. Ilschner, E. J. Kramer, S. Mahajan, P. Veyssire (Eds.), Encyclopedia of Materials: Science and Technology, Elsevier, Oxford, 2011, pp. 1 - 3, current as of 28 October 2015. doi:10.1016/B978-0-08-043152-9.02274-0

URL http://www.sciencedirect.com/science/article/pii/ B9780080431529022740

[7] Y. Zhang, T. T. Zuo, Z. Tang, M. C. Gao, K. A. Dahmen, P. K. Liaw, Z. P. Lu, Microstructures and properties of high-entropy alloys, Prog. Mater. Sci. 61 (2014) 1 - 93. doi:10.1016/j.pmatsci.2013.10.001.

URL http://www.sciencedirect.com/science/article/pii/ S0079642513000789

[8] M.-H. Tsai, J.-W. Yeh, High-entropy alloys: A critical review, Mater. Res. Lett. 2 (3) (2014) 107-123. doi:10.1080/21663831.2014.912690

URL https://doi.org/10.1080/21663831.2014.912690 
[9] E. J. Pickering, N. G. Jones, High-entropy alloys: a critical assessment of their founding principles and future prospects, Int. Mater. Rev. 61 (3) (2016) 183-202. doi:10.1080/09506608.2016.1180020

URL https://doi.org/10.1080/09506608.2016.1180020

[10] M. Laurent-Brocq, L. Perrire, R. Pirs, Y. Champion, From high entropy alloys to diluted multi-component alloys: Range of existence of a solid-solution, Mater. Des. $103 \quad$ (2016) $84 \quad$ - 89. doi:10.1016/j.matdes.2016.04.046.

URL http://www.sciencedirect.com/science/article/pii/ S0264127516305202

[11] F. Otto, Y. Yang, H. Bei, E. George, Relative effects of enthalpy and entropy on the phase stability of equiatomic high-entropy alloys, Acta Mater. 61 (7) (2013) 2628 - 2638. doi:10.1016/j .actamat.2013.01.042 URL http://www.sciencedirect.com/science/article/pii/ S1359645413000694

[12] M. C. Troparevsky, J. R. Morris, P. R. C. Kent, A. R. Lupini, G. M. Stocks, Criteria for predicting the formation of single-phase high-entropy alloys, Phys. Rev. X 5 (2015) 011041. doi:10.1103/PhysRevX.5.011041. URL https ://link .aps.org/doi/10.1103/PhysRevX.5.011041

[13] O. N. Senkov, C. Woodward, D. B. Miracle, Microstructure and properties of aluminum-containing refractory high-entropy alloys, JOM 66 (10) (2014) 2030-2042. doi:10.1007/s11837-014-1066-0. URL https://doi .org/10.1007/s11837-014-1066-0

[14] N. Stepanov, N. Yurchenko, V. Sokolovsky, M. Tikhonovsky, G. Salishchev, An AlNbTiVZr $_{0.5}$ high-entropy alloy combining high specific strength and good ductility, Mater. Lett. 161 (Supplement C) (2015) 136 - 139. doi:10.1016/j.matlet.2015.08.099.

URL http://www.sciencedirect.com/science/article/pii/ S0167577X15304572 
[15] H. Chen, A. Kauffmann, B. Gorr, D. Schliephake, C. Seemller, J. Wagner, H.-J. Christ, M. Heilmaier, Microstructure and mechanical properties at elevated temperatures of a new al-containing refractory high-entropy alloy Nb-Mo-Cr-Ti-Al, J. Alloys Compd. 661 (Supplement C) (2016) 206 - 215. doi:10.1016/j.jallcom.2015.11.050.

URL http://www.sciencedirect.com/science/article/pii/ S0925838815316066

[16] N. Yurchenko, N. Stepanov, S. Zherebtsov, M. Tikhonovsky, G. Salishchev, Structure and mechanical properties of B2 ordered refractory AlNbTiVZr $(x=0-1.5)$ high-entropy alloys, Mater. Sci. Eng. A 704 (Supplement C) (2017) 82 - 90. doi:10.1016/j.msea.2017.08.019

URL http://www.sciencedirect.com/science/article/pii/ S0921509317310249

[17] L.-Y. Tian, G. Wang, J. S. Harris, D. L. Irving, J. Zhao, L. Vitos, Alloying effect on the elastic properties of refractory highentropy alloys, Mater. Des. 114 (Supplement C) (2017) 243 - 252. doi:10.1016/j.matdes.2016.11.079

URL http://www.sciencedirect.com/science/article/pii/ S0264127516314794

[18] O. Senkov, J. Jensen, A. Pilchak, D. Miracle, H. Fraser, Compositional variation effects on the microstructure and properties of a refractory high-entropy superalloy $\mathrm{AlMo}_{0.5} \mathrm{NbTa}_{0.5} \mathrm{TiZr}$, Mater. Des. 139 (2018) 498 - 511. doi:10.1016/j.matdes.2017.11.033.

URL https://www.sciencedirect.com/science/article/pii/ S026412751731064X

[19] N. Sakaguch, M. Niinomi, T. Akahori, Tensile deformation behavior of TiNb-Ta-Zr biomedical alloys, Mater. Trans. 45 (4) (2004) 1113-1119.

[20] R. Banerjee, S. Nag, J. Stechschulte, H. L. Fraser, Strengthening mechanisms in TiNbZrTa and TiMoZrFe orthopaedic alloys, Biomaterials 25 (17) 
(2004) 3413-3419. doi:10.1016/j.biomaterials.2003.10.041.

URL http://www.sciencedirect.com/science/article/pii/ S0142961203009542

[21] G. Yang, T. Zhang, Phase transformation and mechanical properties of the $\mathrm{Ti}_{50} \mathrm{Zr}_{30} \mathrm{Nb}_{10} \mathrm{Ta}_{10}$ alloy with low modulus and biocompatible, J. Alloys Compd. 392 (2005) 291-294. doi:10.1016/j.jallcom.2004.08.099

URL http://www.sciencedirect.com/science/article/pii/ S0925838804011867

[22] Q. Wei, L. Wang, Y. Fu, J. Qin, W. Lu, D. Zhang, Influence of oxygen content on microstructure and mechanical properties of Ti-Nb-Ta-Zr alloy, Mater. Des. 32 (5) (2011) 2934 - 2939. doi:10.1016/j.matdes.2010.11.049.

URL http://www.sciencedirect.com/science/article/pii/ S0261306910006758

[23] V. Brailovski, S. Prokoshkin, M. Gauthier, K. Inaekyan, S. Dubinskiy, M. Petrzhik, M. Filonov, Bulk and porous metastable beta TiNbZr(Ta) alloys for biomedical applications, Mater. Sci. Eng. C 31 (2011) 643-657. doi:10.1016/j.msec.2010.12.008

URL http://www.sciencedirect.com/science/article/pii/ S0928493110003498

[24] M. A.-H. Gepreel, M. Niinomi, Biocompatibility of Ti-alloys for longterm implantation, J. Mech. Behav. Biomed. Mater. 20 (2013) 407-415. doi:10.1016/j.jmbbm.2012.11.014

URL http://www.sciencedirect.com/science/article/pii/ S1751616112003268

[25] F. Tian, L. K. Varga, N. Chen, J. Shen, L. Vitos, Ab initio design of elastically isotropic $\operatorname{TiZrNbMoV}_{x}$ high-entropy alloys J. Alloys Compd. 599 (Supplement C) (2014) $19 \quad-25$. doi:10.1016/j.jallcom.2014.01.237. 
URL

http://www.sciencedirect.com/science/article/pii/ S0925838814003089

[26] V. Nguyen, M. Qian, Z. Shi, T. Song, L. Huang, J. Zou, A novel quaternary equiatomic Ti-Zr-Nb-Ta medium entropy alloy (MEA), Intermetallics 101 (2018) 39 - 43. doi:https: //doi.org/10.1016/j.intermet.2018.07.008.

URL http://ww.sciencedirect.com/science/article/pii/ S0966979518304655

[27] M. Todai, T. Nagase, T. Hori, A. Matsugaki, A. Sekita, T. Nakano, Novel TiNbTaZrMo high-entropy alloys for metallic biomaterials, Scr. Mater. 129 (2017) 65-68. doi:10.1016/j.scriptamat.2016.10.028

URL http://ww.sciencedirect.com/science/article/pii/ S1359646216305231

[28] S.-P. Wang, J. Xu, TiZrNbTaMo high-entropy alloy designed for orthopedic implants: As-cast microstructure and mechanical properties, Mater. Sci. Eng. C 73 (2017) 80-89. doi:10.1016/j.msec.2016.12.057.

URL http://www.sciencedirect.com/science/article/pii/ S0928493116326339

[29] I. Milošev, CoCrMo Alloy for Biomedical Applications, Vol. 55, Springer US, Boston, MA, 2012, pp. 1-72. doi:10.1007/978-1-4614-3125-1_1.

URL https ://doi .org/10.1007/978-1-4614-3125-1_1

[30] B. L. Gyorffy, Coherent-potential approximation for a nonoverlappingmuffin-tin-potential model of random substitutional alloys, Phys. Rev. B 5 (1972) 2382-2384. doi:10.1103/PhysRevB.5.2382.

URL https://link.aps.org/doi/10.1103/PhysRevB.5.2382

[31] P. Soven, Coherent-potential model of substitutional disordered alloys Phys. Rev. 156 (1967) 809-813. doi:10.1103/PhysRev.156.809. URL https://link .aps.org/doi/10.1103/PhysRev .156.809 
[32] F. Tian, L. K. Varga, N. Chen, L. Delczeg, L. Vitos, Ab initio investigation of high-entropy alloys of $3 d$ elements, Phys. Rev. B 87 (2013) 075144. doi: 10.1103/PhysRevB.87.075144.

URL https://link.aps.org/doi/10.1103/PhysRevB.87.075144

[33] D. Ma, B. Grabowski, F. Körmann, J. Neugebauer, D. Raabe, Ab initio thermodynamics of the CoCrFeMnNi high entropy alloy: Importance of entropy contributions beyond the configurational one, Acta Mater. 100 (2015) 90 - 97. doi:10.1016/j.actamat.2015.08.050.

URL http://www.sciencedirect.com/science/article/pii/ S1359645415006278

[34] A. Zunger, S.-H. Wei, L. G. Ferreira, J. E. Bernard, Special quasirandom structures, Phys. Rev. Lett. 65 (1990) 353-356. doi:10.1103/ PhysRevLett.65.353

URL https://link.aps.org/doi/10.1103/PhysRevLett.65.353

[35] S.-H. Wei, L. G. Ferreira, J. E. Bernard, A. Zunger, Electronic properties of random alloys: Special quasirandom structures, Phys. Rev. B 42 (1990) 9622-9649. doi:10.1103/PhysRevB.42.9622.

URL https://link.aps.org/doi/10.1103/PhysRevB.42.9622

[36] A. J. Zaddach, C. Niu, C. C. Koch, D. L. Irving, Mechanical properties and stacking fault energies of NiFeCrCoMn high-entropy alloy, JOM 65 (12) (2013) 1780-1789. doi:10.1007/s11837-013-0771-4.

URL https://doi .org/10.1007/s11837-013-0771-4

[37] H. S. Oh, D. Ma, G. P. Leyson, B. Grabowski, E. S. Park, F. Körmann, D. Raabe, Lattice distortions in the FeCoNiCrMn high entropy alloy studied by theory and experiment, Entropy 18 (9).

URL http://www.mdpi .com/1099-4300/18/9/321

[38] A. R. Oganov, C. W. Glass, Crystal structure prediction using ab initio evolutionary techniques: Principles and applications, J. Chem. Phys. 124 
(2006) 244704. doi:10.1063/1.2210932.

URL https://doi.org/10.1063/1.2210932

[39] A. R. Oganov, H. Stokes, M. Valle, How evolutionary crystal structure prediction works - and why. Acc. Chem. Res. 44 (3) (2011) 227-237. doi : 10.1021/ar1001318

URL https://doi.org/10.1021/ar1001318

[40] A. O. Lyakhov, A. R. Oganov, H. Stokes, Q. Zhu, New developments in evolutionary structure prediction algorithm USPEX, Comp. Phys. Comm.

184 (4) (2013) 1172-1182. doi:10.1016/j.cpc.2012.12.009

URL http://wWw.sciencedirect.com/science/article/pii/ S0010465512004055

[41] A. R. Oganov, Modern Methods of Crystal Structure Prediction, WileyVCH, Berlin, 2010.

[42] G. Kresse, J. Furthmüller, Efficient iterative schemes for ab initio totalenergy calculations using a plane-wave basis set, Phys. Rev. B 54 (1996) 11169-11186. doi:10.1103/PhysRevB.54.11169

URL https://link.aps .org/doi/10.1103/PhysRevB.54.11169

[43] G. Kresse, J. Furthmüller, Efficiency of ab-initio total energy calculations for metals and semiconductors using a plane-wave basis set, Comp. Mat. Sci. 6 (1) (1996) 15 - 50 . doi:http: //dx.doi.org/10.1016/0927-0256(96)00008-0. URL http://www.sciencedirect.com/science/article/pii/ 0927025696000080

[44] A. R. Oganov, M. Valle, How to quantify energy landscapes of solids, The Journal of Chemical Physics 130 (10) (2009) 104504. arXiv:https://doi. org/10.1063/1.3079326, doi:10.1063/1.3079326

URL https://doi.org/10.1063/1.3079326 
[45] S. Guo, C. Ng, J. Lu, C. T. Liu, Effect of valence electron concentration on stability of fcc or bcc phase in high entropy alloys, J. Appl. Phys. 109 (10) (2011) 103505. doi:10.1063/1.3587228.

URL http://dx.doi.org/10.1063/1.3587228

[46] Y. Le Page, P. Saxe, Symmetry-general least-squares extraction of elastic data for strained materials from ab initio calculations of stress, Phys. Rev.

B 65 (2002) 104104. doi:10.1103/PhysRevB.65.104104

URL https://link.aps.org/doi/10.1103/PhysRevB.65.104104

[47] N. W. Ashcroft, N. D. Mermin, Solid State Physics, Saunders College, Philadelphia, 1976.

[48] C. Kittel, Introduction to Solid State Physics, 6th Edition, John Wiley \& Sons, Inc., New York, 1986.

[49] J. F. Nye, Physical Properties of Crystals, Oxford University Press, 1985.

[50] F. Tasnádi, M. Odén, I. A. Abrikosov, Ab initio elastic tensor of cubic $\mathrm{Ti}_{0.5} \mathrm{Al}_{0.5} \mathrm{~N}$ alloys: Dependence of elastic constants on size and shape of the supercell model and their convergence, Phys. Rev. B 85 (2012) 144112. doi:10.1103/PhysRevB.85.144112

URL https://link.aps.org/doi/10.1103/PhysRevB.85.144112

[51] M. C. Gao, Y. Suzuki, H. Schweiger, Ö. N. Doğan, J. Hawk, M. Widom, Phase stability and elastic properties of Cr-V alloys, J. Phys. Condens. Matter 25 (7) (2013) 075402.

URL http://stacks .iop.org/0953-8984/25/i=7/a=075402

[52] K. W. Andrews, Elastic moduli of polycrystalline cubic metals, J. Phys. D: Appl. Phys. 11 (18) (1978) 2527.

URL http://stacks .iop.org/0022-3727/11/i=18/a=011

[53] W. Voigt, Lehrbuch der Kristallphysik, Taubner, Leipzig, 1928. 
[54] A. Reuss, Berechnung der fließgrenze von mischkristallen auf grund der plastizitätsbedingung für einkristalle, J. Appl. Math. Mech. 9 (1) (1929) $49-58$.

[55] R. Hill, The elastic behaviour of a crystalline aggregate, Proc. Phys. Soc. A 65 (5) (1952) 349.

URL http://stacks .iop.org/0370-1298/65/i=5/a=307

[56] C. Zener, Elasticity and anelasticity of metals, Chicago, Illinois : University of Chicago Press, 1948.

[57] J. P. Perdew, K. Burke, M. Ernzerhof, Generalized gradient approximation made simple, Phys. Rev. Lett. 77 (1996) 3865-3868. doi:10.1103/ PhysRevLett.77.3865.

URL http://link.aps.org/doi/10.1103/PhysRevLett.77.3865

[58] P. E. Blöchl, Projector augmented-wave method, Phys. Rev. B 50 (1994) 17953. doi:10.1103/PhysRevB.50.17953.

[59] G. Kresse, D. Joubert, From ultrasoft pseudopotentials to the projector augmented-wave method, Phys. Rev. B 59 (1999) 1758-1775. doi:10. 1103/PhysRevB.59.1758.

URL http://link.aps.org/doi/10.1103/PhysRevB.59.1758

[60] P. E. Blöchl, O. Jepsen, O. K. Andersen, Improved tetrahedron method for brillouin-zone integrations, Phys. Rev. B 49 (1994) 16223-16233. doi: 10.1103/PhysRevB.49.16223

URL https://link.aps.org/doi/10.1103/PhysRevB.49.16223

[61] M. Methfessel, A. T. Paxton, High-precision sampling for brillouin-zone integration in metals, Phys. Rev. B 40 (1989) 3616-3621. doi:10.1103/ PhysRevB.40.3616.

URL http://link.aps.org/doi/10.1103/PhysRevB.40.3616

[62] H. J. Monkhorst, J. D. Pack, Special points for brillouin-zone integrations, Phys. Rev. B 13 (1976) 5188. doi:10.1103/PhysRevB.13.5188. 
[63] L. Schimka, R. Gaudoin, J. Klimeš, M. Marsman, G. Kresse, Lattice constants and cohesive energies of alkali, alkaline-earth, and transition metals: Random phase approximation and density functional theory results, Phys.

Rev. B 87 (2013) 214102. doi:10.1103/PhysRevB.87.214102.

URL https://link .aps.org/doi/10.1103/PhysRevB.87.214102

[64] P. Janthon, S. A. Luo, S. M. Kozlov, F. Viñes, J. Limtrakul, D. G. Truhlar, F. Illas, Bulk properties of transition metals: A challenge for the design of universal density functionals, J. Chem Theory Comput. 10 (9) (2014) 3832-3839. doi:10.1021/ct500532v.

[65] F. Cardarelli, Materials Handbook, 2nd Edition, Springer-Verlag, 2008, p. 268.

[66] L. Koči, Y. Ma, A. R. Oganov, P. Souvatzis, R. Ahuja, Elasticity of the superconducting metals $\mathrm{V}, \mathrm{Nb}$, Ta, Mo, and $\mathrm{W}$ at high pressure, Phys. Rev. B 77 (2008) 214101. doi:10.1103/PhysRevB.77.214101. URL https://link.aps.org/doi/10.1103/PhysRevB.77.214101

[67] L. Zenghui, S. Jiaxiang, First principles calculations of electronic properties and mechanical properties of bcc molybdenum and niobium, Rare Metals 30 (2011) 354. doi:10.1007/s12598-011-0302-9.

[68] C. Kittel, Introduction to Solid State Physics, 8th Edition, John Wiley \& Sons, Inc., New York, 2005.

[69] K. Momma, F. Izumi, VESTA3 for three-dimensional visualization of crystal, volumetric and morphology data, J. Appl. Crystallogr. 44 (6) (2011) 1272-1276. doi:10.1107/S0021889811038970.

[70] Note that since the lattices are slightly distorted, the $a$-values in the table are actually mean values and the errors correspond to the standard deviations.

[71] S.-P. Wang, J. Xu, (TiZrNbTa)-Mo high-entropy alloys: Dependence of microstructure and mechanical properties on Mo concentration and 
modeling of solid solution strengthening, Intermetallics 95 (2018) $59-72$.

doi:10.1016/j.intermet.2018.01.017.

URL http://www.sciencedirect.com/science/article/pii/

S0966979517310440

[72] H. Ge, F. Tian, Y. Wang, Elastic and thermal properties of refractory highentropy alloys from first-principles calculations, Computational Materials Science 128 (2017) 185 - 190. doi:10.1016/j.commatsci.2016.11.035.

URL http://wwW.sciencedirect.com/science/article/pii/ S0927025616305961

[73] W. Oliver, G. Pharr, An improved technique for determining hardness and elastic modulus using load and displacement sensing indentation experiments, J. Materials Research 7 (6) (1992) 15641583. doi:10.1557/JMR. 1992.1564.

[74] S. Qiu, N. Miao, J. Zhou, Z. Guo, Z. Sun, Strengthening mechanism of aluminum on elastic properties of NbVTiZr high-entropy alloys, Intermetallics 92 (2018) 7 -14. doi:10.1016/j.intermet.2017.09.003.

URL http://www.sciencedirect.com/science/article/pii/ S0966979517307070

[75] Y. Tian, B. Xu, Z. Zhao, Microscopic theory of hardness and design of novel superhard crystals, Int. J. Refract. Metals Hard Mater. 33 (2012) 93

- 106. doi:10.1016/j.ijrmhm.2012.02.021.

URL http://www.sciencedirect.com/science/article/pii/ S026343681200042X

[76] S. Pugh, XCII. Relations between the elastic moduli and the plastic properties of polycrystalline pure metals, Lond. Edinb. Phil. Mag. 45 (367) (1954) 823-843. doi:10.1080/14786440808520496.

URL http://dx.doi .org/10.1080/14786440808520496

[77] Y. Ikeda, B. Grabowski, F. Körmann, Ab initio phase stabilities and mechanical properties of multicomponent alloys: A comprehensive review 
for high entropy alloys and compositionally complex alloys, Mater. Charact.doi:10.1016/j.matchar.2018.06.019.

URL http://www.sciencedirect.com/science/article/pii/ S1044580318306636

[78] J. J. Lewandowski, W. H. Wang, A. L. Greer, Intrinsic plasticity or brittleness of metallic glasses, Philos. Mag. Lett. 85 (2) (2005) 77-87. doi:10.1080/09500830500080474.

URL http://dx.doi.org/10.1080/09500830500080474

[79] D. G. Pettifor, Theoretical predictions of structure and related properties of intermetallics, Mater. Sci. Tech. 8 (4) (1992) 345-349. arXiv:https:// doi.org/10.1179/mst.1992.8.4.345, doi:10.1179/mst.1992.8.4.345 URL https://doi.org/10.1179/mst.1992.8.4.345

[80] D. Nguyen-Manh, M. Mrovec, S. P. Fitzgerald, Dislocation driven problems in atomistic modelling of materials, Mater. Trans. 49 (11) (2008) 2497-2506. doi:10.2320/matertrans.MB200827.

[81] U. Aydin, H. Titrian, M. Friák, Self-consistent calculations - elasticity of multi-phase aggregates, Duesseldorf, Germany (2012-2013).

URL http://scema.mpie.de/

[82] H. Titrian, U. Aydin, M. Friák, D. Ma, D. Raabe, J. Neugebauer, Selfconsistent scale-bridging approach to compute the elasticity of multi-phase polycrystalline materials, MRS Proceedings 1524. doi:10.1557/opl. 2013.41.

[83] M. Friák, W. A. Counts, D. Ma, B. Sander, D. Holec, D. Raabe, J. Neugebauer, Theory-guided materials design of multi-phase Ti-Nb alloys with bone-matching elastic properties, Materials 5 (10) (2012) 1853-1872. doi: $10.3390 / \mathrm{ma} 5101853$

URL http://www.mdpi.com/1996-1944/5/10/1853 
[84] L.-F. Zhu, M. Friák, L. Lymperakis, H. Titrian, U. Aydin, A. Janus, H.-O. Fabritius, A. Ziegler, S. Nikolov, P. Hemzalová, D. Raabe, J. Neugebauer, $\mathrm{Ab}$ initio study of single-crystalline and polycrystalline elastic properties of Mg-substituted calcite crystals, J. Mech. Behav. Biomed. Mater. 20 (Supplement C) (2013) 296 - 304. doi:10.1016/j.jmbbm.2013.01.030

URL http://wWw.sciencedirect.com/science/article/pii/ S1751616113000465

[85] R. Dronskowski, P. E. Blöchl, Crystal orbital hamilton populations (COHP). energy-resolved visualization of chemical bonding in solids based on density-functional calculations, J. Phys. Chem. 97 (1993) 8617-8624. doi:10.1021/j100135a014.

URL https://doi.org/10.1021/j100135a014

[86] S. Maintz, V. L. Deringer, M. Esser, R. Nelson, A. Tchougréeff, R. Dronskowski, Local orbital basis suite towards electronic-structure reconstruction, Aachen, Germany (2010-2017).

URL http://www. cohp.de/

[87] V. L. Deringer, A. L. Tchougréeff, R. Dronskowski, Crystal orbital hamilton population (COHP) analysis as projected from plane-wave basis sets, J. Phys. Chem. A 115 (2011) 5461-5466. doi:10.1021/jp202489s

URL https://doi.org/10.1021/jp202489s

[88] S. Maintz, L. D. V, A. L. Tchougréeff, R. Dronskowski, Analytic projection from plane-wave and paw wavefunctions and application to chemicalbonding analysis in solids, J. Comput. Chem. 34 (29) (2013) 2557-2567. doi:10.1002/jcc.23424.

URL http://dx.doi.org/10.1002/jcc.23424

[89] S. Maintz, V. L. Deringer, A. L. Tchougreff, R. Dronskowski, LOBSTER: A tool to extract chemical bonding from plane-wave based dft, J. Comp. Chem. 37 (11) (2016) 1030-1035. doi:10.1002/jcc.24300.

URL http://dx.doi.org/10.1002/jcc.24300 
[90] C. D. Gelatt, A. R. Williams, V. L. Moruzzi, Theory of bonding of transition metals to nontransition metals, Phys. Rev. B 27 (1983) 2005-2013. doi:10.1103/PhysRevB.27.2005.

URL https://link.aps.org/doi/10.1103/PhysRevB.27.2005

[91] P. Ravindran, R. Asokamani, Correlation between electronic structure, mechanical properties and phase stability in intermetallic compounds, Bull. Mater. Sci. 20 (4) (1997) 613-622. doi:10.1007/BF02744780.

URL https://doi.org/10.1007/BF02744780

[92] Z. Wu, X. Hao, X. Liu, J. Meng, Structures and elastic properties of Osn 2 investigated via first-principles density functional calculations, Phys. Rev. B 75 (2007) 054115. doi:10.1103/PhysRevB.75.054115.

URL https://link.aps.org/doi/10.1103/PhysRevB.75.054115

[93] G. A. Landrum, R. Dronskowski, The orbital origins of magnetism: From atoms to molecules to ferromagnetic alloys, Angew. Chem. Int. Ed. 39 (9) (2000) 1560-1585. doi:10.1002/(SICI) 1521-3773(20000502)39: 9<1560: :AID-ANIE1560>3.0.C0;2-T

URL http://dx.doi.org/10.1002/(SICI)1521-3773(20000502)39: 9<1560: :AID-ANIE1560>3.0.CD;2-T 